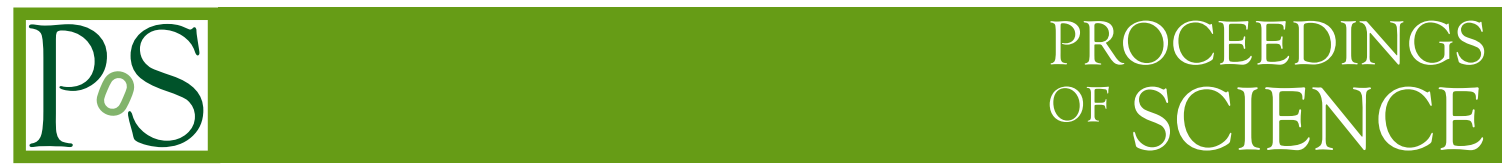

\title{
Recent lattice QCD results on nucleon structure
}

\section{Konstantinos Orginos*}

The College of William and Mary, Department. of Physics, Williamsburg VA 23187-8795, USA. Jefferson Lab, MS 12H2, 12000 Jefferson Av., Newport News, VA 23606, USA.

E-mail: kostas@wm. edu

I review recent developments in lattice calculations of nucleon structure. In particular, I cover the calculations of nucleon matrix elements related to generalized parton distribution functions, structure functions and form factors.

XXIV International Symposium on Lattice Field Theory

July 23-28 2006

Tucson Arizona, US

${ }^{*}$ Speaker. 


\section{Introduction}

One of the major tasks of lattice QCD is computing the properties of hadrons form the underlying theory of QCD. Over the last several years significant effort has been devoted into lattice calculation of the nucleon structure. Traditionally, nucleon structure has been probed in both elastic scattering experiments, where form factors are measured, and deep inelastic scattering experiments, where nucleon structure functions are measured. Recently, hard exclusive processes have been used to define the so called generalized parton distribution functions (GPD) $[1,2,3,4,5,6,7,8,9]$ which encompass both form factors and structure functions in certain limits. For that reason, lattice calculations of the GPDs have been one of the major efforts to understand nucleon structure from lattice QCD during the last few years.

The generalized parton distribution functions are defined by off-forward matrix elements of bilocal operators on the light cone

$$
\mathscr{O}_{\Gamma}(x)=\int \frac{d \lambda}{4 \pi} e^{i \lambda x} \bar{q}\left(\frac{-\lambda n}{2}\right) \Gamma \mathscr{P} e^{-i g \int_{-\lambda / 2}^{\lambda / 2} d \alpha n \cdot A(\alpha n)} q\left(\frac{\lambda n}{2}\right),
$$

where $x$ is the longitutinal momentum fraction, $n$ is a light cone vector, $\Gamma$ could be $\Gamma=\not h, \not h \gamma_{5}$ or $\Gamma=n_{\mu} \sigma^{\mu v} \gamma_{5}$. If we define $t=-\Delta^{2}$, with $\Delta=P^{\prime}-P$ the 4-momentum transfer, and $\xi=-n \cdot \Delta / 2$, the longitudinal momentum transfer, these matrix elements can be parameterized in terms of nine independent generalized distribution functions:

$$
\begin{aligned}
&\left\langle P, s\left|\mathscr{O}_{\eta \prime}(x)\right| P^{\prime}, s^{\prime}\right\rangle=\bar{u}(p, s)\left[\not h H(x, \xi, t)+\frac{n_{\mu} \Delta_{v}}{2 m} i \sigma^{\mu v} E(x, \xi, t)\right] u\left(p^{\prime}, s^{\prime}\right) \\
&\left\langle P, s\left|\mathscr{O}_{\eta / \gamma_{5}}(x)\right| P^{\prime}, s^{\prime}\right\rangle=\bar{u}(p, s)\left[\not h \gamma_{5} \tilde{H}(x, \xi, t)+\frac{n \cdot \Delta}{2 m} \gamma_{5} \tilde{E}(x, \xi, t)\right] u\left(p^{\prime}, s^{\prime}\right) \\
&\left\langle P, s\left|\mathscr{O}_{5 T}(x)\right| P^{\prime}, s^{\prime}\right\rangle=\bar{u}(p, s)\left[n_{\mu} \sigma^{\mu k} \gamma_{5}\left(H_{T}(x, \xi, t)-\frac{t}{2 m^{2}} \tilde{H}_{T}\right)+\right. \\
&+\frac{\varepsilon^{\mu v \alpha \beta} \Delta_{\alpha} \gamma_{\beta}}{2 m}\left(E_{T}(x, \xi, t)+2 \tilde{H}_{T}(x, \xi, t)\right)+ \\
&\left.+\frac{n_{\mu} \Delta^{[\mu} \sigma^{v] \alpha} \gamma_{5} \Delta_{\alpha}}{2 m^{2}} \tilde{H}_{T}(x, \xi, t)+\frac{\varepsilon^{\mu v \alpha \beta} P_{\alpha} \gamma_{\beta}}{m} \tilde{E}_{T}(x, \xi, t)\right] u\left(p^{\prime}, s^{\prime}\right)
\end{aligned}
$$

In the forward limit, i.e. $\Delta=0$ the regular parton distribution functions such as the quark density, helicity and transversity distributions are recovered:

$$
\begin{aligned}
H(x, 0,0) & =q(x) \\
\tilde{H}(x, 0,0) & =\Delta q(x) \\
H_{T}(x, 0,0) & =\delta q(x) .
\end{aligned}
$$

The first moments of the GPDs are the regular form factors. For example the vector form factors:

$$
\begin{aligned}
& \int_{-1}^{1} d x H(x, \xi, t)=F_{1}(t) \\
& \int_{-1}^{1} d x E(x, \xi, t)=F_{2}(t)
\end{aligned}
$$



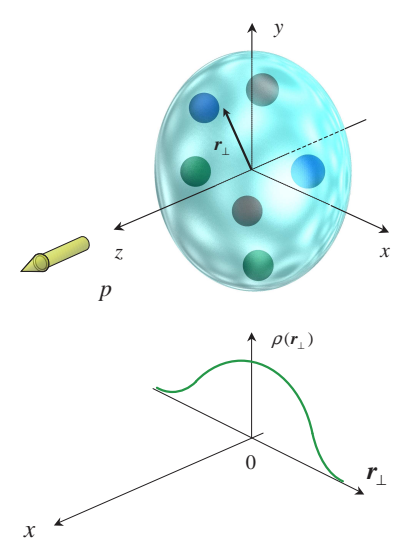
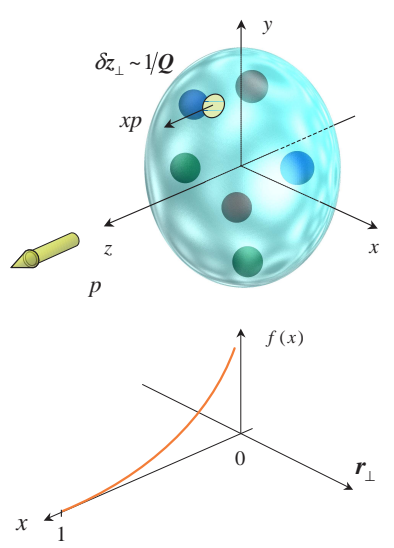
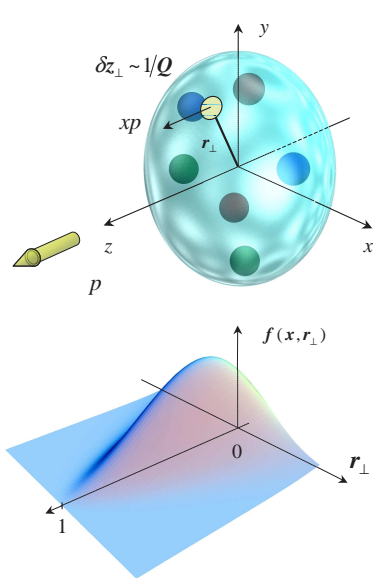

Figure 1: The infinite momentum frame probabilistic interpretation of form factors (left), parton distributions (center), and generalized parton distribution functions at $\xi=0$ (right).

and the axial vector form factors.

$$
\begin{gathered}
\int_{-1}^{1} d x \tilde{H}(x, \xi, t)=g_{A}(t) \\
\int_{-1}^{1} d x \tilde{E}(x, \xi, t)=g_{P}(t)
\end{gathered}
$$

In the infinite momentum frame and $\xi=0$ the generalized parton distribution functions admit a very simple probabilistic interpretation (see Fig. $1^{1}$ ) $[7,10,11,12,13,14,15]$. By taking the inverse Fourier transform with respect to the transverse momentum transfer $\Delta_{\perp}$, we obtain distributions in longitudinal momentum fraction and impact parameter space. For example

$$
\int \frac{d^{2} \Delta_{\perp}}{\left(2 \pi^{2}\right)^{2}} e^{-i \Delta_{\perp} \cdot r_{\perp}} H(x, 0, t)=f\left(x, r_{\perp}\right)
$$

where $r_{\perp}$ is the impact parameter vector living on the two dimensional transverse space. Taking the first moment of $f\left(x, r_{\perp}\right)$, i.e. integrating over the momentum fraction $x$, we obtain the charge distribution $\rho\left(r_{\perp}\right)$ in impact parameter space. In the forward limit, i.e. no momentum transfer, $f\left(x, r_{\perp}\right)$ becomes the quark density $f(x)$ with momentum fraction $x$. In general $f\left(x, r_{\perp}\right)$ is the probability to find a parton with momentum fraction $x$ at the point $r_{\perp}$ in the transverse plane. As it is shown in Fig 1(right most panel), the function $f\left(x, r_{\perp}\right)$ becomes increasingly narrow and small as $x$ approaches one. This is just due to the fact that the probability of finding a single constituent carrying all the momentum of the nucleon is negligible.

Lattice QCD can only contribute to the calculation of the moments of these objects. The moments are related to nucleon matrix elements of local operators. These matrix elements can be rotated to Euclidean space and then computed on the lattice. The moments of GPDs are related to matrix elements of simple quark bi-linear operators,

$$
\left\langle p^{\prime}, s^{\prime}\left|\mathscr{O}_{\mu_{1} \mu_{2} \cdots \mu_{n}}^{\gamma}\right| p, s\right\rangle
$$

\footnotetext{
${ }^{1}$ Figure taken from [9].
} 
where the operators $\mathscr{O}_{\mu_{1} \mu_{2} \cdots \mu_{n}}^{\gamma}$ are given by

$$
\begin{aligned}
\mathscr{O}_{\mu_{1} \mu_{2} \cdots \mu_{n}}^{q} & =\left(\frac{i}{2}\right)^{n-1} \bar{q} \gamma_{\mu_{1}} \stackrel{\leftrightarrow}{D}_{\mu_{2}} \cdots \stackrel{\leftrightarrow}{D}_{\mu_{n}} q \text {-trace }, \\
\mathscr{O}_{\sigma \mu_{1} \mu_{2} \cdots \mu_{n}}^{5 q} & =\left(\frac{i}{2}\right)^{n-1} \bar{q} \gamma_{\sigma} \gamma_{5} \stackrel{\leftrightarrow}{D}_{\mu_{2}} \cdots \stackrel{\leftrightarrow}{D}_{\mu_{n}} q-\text { trace } \\
\mathscr{O}_{\rho v \mu_{1} \mu_{2} \cdots \mu_{n}}^{\sigma q} & =\left(\frac{i}{2}\right)^{n} \bar{q} \sigma_{\rho v} \stackrel{\leftrightarrow}{D}_{\mu_{1}} \cdots \stackrel{\leftrightarrow}{D}_{\mu_{n}} q-\text { trace } \\
\mathscr{O}_{\rho v \mu_{1} \mu_{2} \cdots \mu_{n}}^{\sigma 5 q} & =\left(\frac{i}{2}\right)^{n} \bar{q} \gamma_{5} \sigma_{\rho v} \stackrel{\leftrightarrow}{D}_{\mu_{1}} \cdots \stackrel{\leftrightarrow}{D}_{\mu_{n}} q-\text { trace }
\end{aligned}
$$

and $\stackrel{\leftrightarrow}{D}=\vec{D}-\overleftarrow{D}$ and $\vec{D}, \overleftarrow{D}$ are covariant derivatives acting on the right and the left, respectively The indices are symmetrized, and in some cases antisymmetrized. The moments of the regular parton distribution functions are then related to the forward limit of the above matrix elements. In addition, the matrix elements with no derivatives define the elastic axial vector and vector form factors of the nucleon. The above matrix elements define the generalized from factors. The moments of the GPDs are polynomials in $\xi$, with coefficients given by generalized form factors, which can be easily computed on the lattice. Standard techniques of extracting matrix elements from three point functions apply. However, over the last several years it has been realized that getting results that can be of physical relevance is a rather non-trivial task. Careful study of all the systematics of the lattice calculation is required. In the last few years though we have been making steady progress in accounting for all these systematics and obtaining results that are very encouraging.

\section{Lattice Actions}

One of the major problems in hadron structure calculations on the lattice is the approach to the physical pion mass. During the last few years developments in both the algorithms and the lattice actions used allowed us to make significant progress in this area. For realistic lattice calculations dynamical fermions with pion masses below $400 \mathrm{MeV}$ are needed. This allows chiral extrapolation formulas to be used with some reliability. In addition a dynamical strange quark is required in order to guarantee that the low energy constants of the Chiral Lagrangian used in the chiral extrapolations match those of the physical theory. Large physical volumes are also needed so that finite volume systematic errors are under control. These constrains make realistic lattice calculations possible only very recently.

The development of actions with exact chiral symmetry on the lattice has been proven to be a significant help. The first quenched lattice calculations with such actions have already been done with pion masses significantly lighter than those used before $[16,17,18]$. Unfortunately, the cost of these actions is such that only very recently have the first results with two light dynamical flavors appeared [19, 20].

The development of improved Kogut-Susskind fermions was a significant step towards light dynamical fermion calculations [21, 22, 23, 24, 25]. Recent work by the LHP collaboration takes advantage of both developments, working with pion masses as low as $300 \mathrm{MeV}$ [26, 27, 28, 29]. The Kogut-Susskind fermions provide a cheap representation of the quark loop effects, while the 
use of domain wall fermions in the valence sector takes advantage of the constraints of chiral symmetry in both the renormalization of the operators and the effective field theory need to extrapolate to the chiral point. Although one might think that this mixed action scheme is a complication difficult to control, in practice it has been shown that the effects of the miss match between the sea and the valence sectors are small in the case of flavor non-singlet quantities. This is very clearly seen in the work by NPLQCD [30, 31, 32, 33, 34, 35]. In theory all these complications can be taken care of in the context of mixed action chiral perturbation theory [36, 37]. One problem with KogutSusskind fermions is that there is still a theoretical issue of the validity of computations when the number of flavors is not an integer multiple of four, but recent theoretical work indicates that the troublesome non-localities of the lattice action go away in the continuum limit [38, 39, 40, 41]. Hence these effects are most likely taken care of in the continuum limit.

Recently, algorithmic developments [42, 43, 44, 45, 46, 47, 48, 49, 50] together with increased computational power, has allowed for dynamical Wilson and improved Wilson fermion calculations. The QCDSF collaboration is using such a formulation to achieve pion masses below 300 $\mathrm{MeV}$.

Finally, the development of twisted mass Wilson fermions opens up yet an other avenue for approaching the chiral limit $[51,52,53,54,55,56,57]$. The ETM collaboration has applied this technology to the moments of pion structure functions with pion masses as low as $250 \mathrm{MeV}[58$, 53].

\section{Renormalization}

One of the main problems we face in calculating the moments of GPDs is the renormalization of lattice operators. The fact that the full $\mathrm{O}(4)$ Euclidean rotational symmetry is broken down to the hypercubic $\mathrm{H}(4)$ symmetry results in operator mixings that are not allowed in the continuum. In particular, for higher moments, the mixing with lower dimensional operators cannot be avoided, limiting us to the ability to compute only the first three moments. Recently Detmold and Lin proposed a method to avoid this problem [59]. However, it remains to be decided how practical this idea is.

The use of fermions with chiral symmetry on the lattice eliminates in some cases, such as the $d_{n}$ matrix elements related to the moments of the polarized structure functions, mixings with lower dimensional operators. An example of this can be seen in [17].

Perturbation theory has been widely used in computing the renormalization constants. Recently Gockeler et al. [60,61] have revisited the issue of operator mixing and renormalization of twist two operators for Wilson and Clover Wilson fermions. In the case of off-forward matrix elements the mixing pattern is more complicated than in the case of forward matrix elements hence a more careful study is required. In addition, perturbative calculations for the case of the Neuberger overlap operator for several choices of the gauge action have been done by Ioannou and Panagopoulos [62], and for the case of domain wall fermions with by Bistrovic [63] and Capitani et al. [64].

Finally, QCDSF and RBC collaborations have used successfully the Rome-Southhampton non-perturbative renormalization technique $[65,66,17,67]$. 


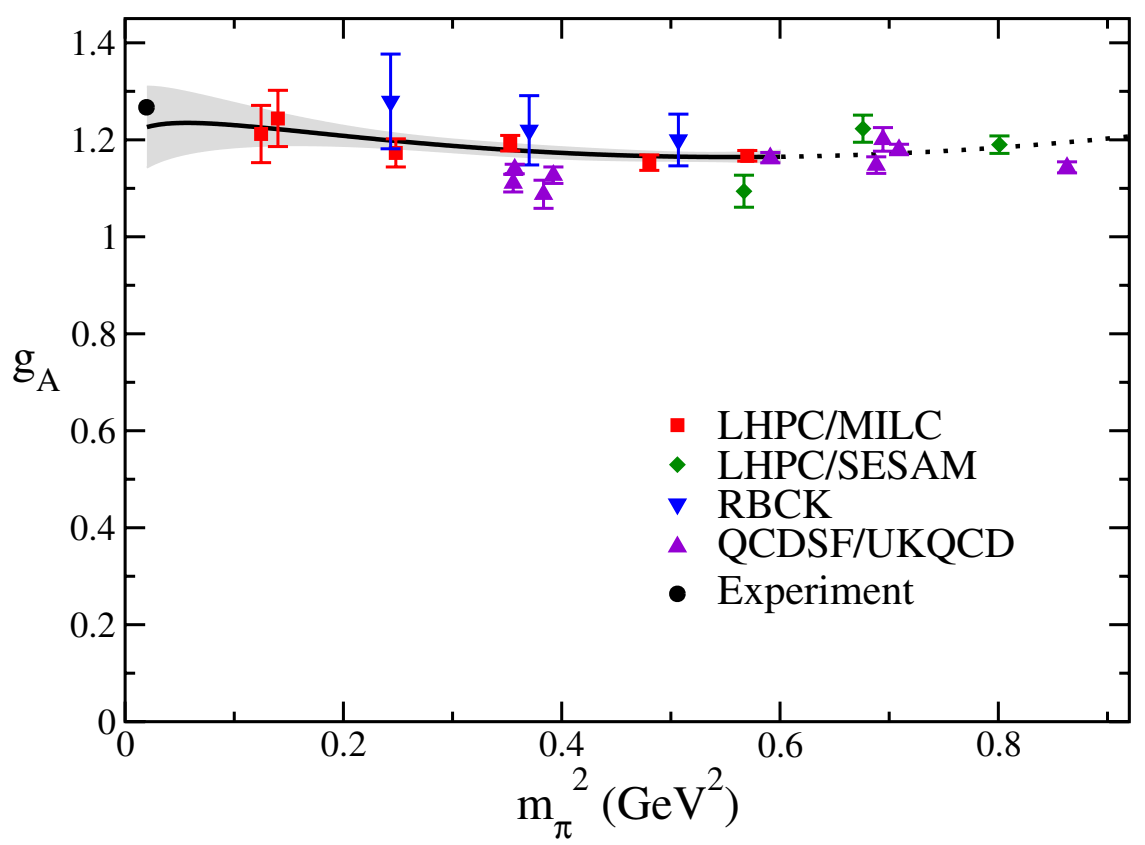

Figure 2: Lattice calculations of the nucleon axial coupling.

\section{Axial coupling of the proton}

One of the most accurately known quantities related to nucleon structure is the nucleon axial coupling. It can be very accurately measured in neutron beta decay experiments. Its deviation from one is a direct indication of the non-trivial structure of the nucleon. The latest particle data book [68] value is

$$
\frac{g_{A}}{g_{V}}=1.2695(29)
$$

It is very difficult to compute this number on the lattice to such a precision. In addition to other systematics, one needs to properly account for charge symmetry breaking effects. However, computing this number from lattice QCD would be a significant success. Present calculations can compute it to an accuracy a little bit better than $10 \%$. One still relies on chiral extrapolations from rather heavy pion masses. As a result the true systematic error due to the chiral extrapolation is difficult to quantify. It is remarkable that over a wide range of pion masses very little dependence of the quark mass is observed.

One of the recent interesting results is the fact that the axial coupling seems to be sensitive to finite volume corrections. One of the first studies that observed this was a quenched domain wall fermion calculation [69]. Recently, the finite volume effect has also been observed in two flavor dynamical improved Wilson fermion calculations by QCDSF [70, 71]. Their results, although at relatively heavy pion masses, corrected for finite volume effects, resulting in an extrapolated number compatible with experiment within the quoted statistical error. QCDSF has also reported some preliminary results at significantly lighter pion masses. 


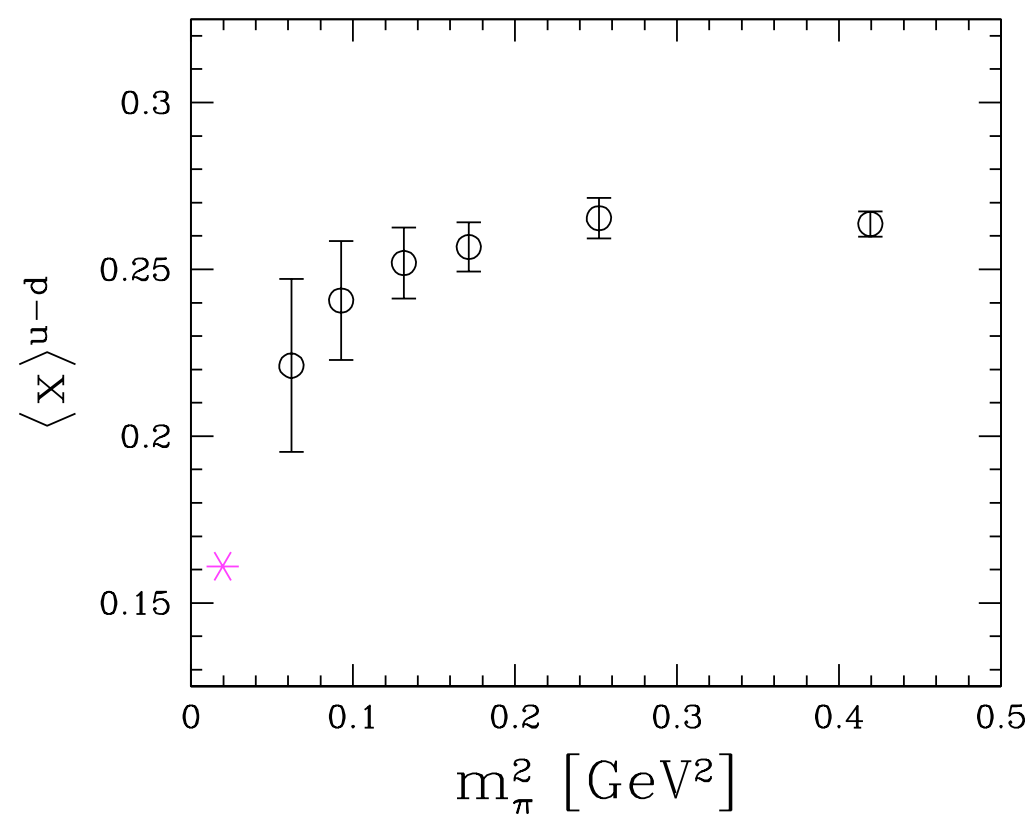

Figure 3: QCDSF results for the momentum fraction computed with overlap fermions in the quenched approximation.

LHPC has computed $g_{A}$ using the mixed action scheme (domain wall valence on KogutSusskind sea) at one lattice spacing with lightest pion mass $350 \mathrm{MeV}$. The results corrected for finite volume using [72] are presented in Fig. 2. The result of the chiral extrapolation using the one loop chiral perturbation theory including the Delta resonance [72] is $g_{A}=1.22(8)$ which is consistent with experiment within errors. Recent work by Bernard and Meissner [73], going to two loop chiral perturbation theory, indicates that one may need pion masses below $300 \mathrm{MeV}$ before reliable chiral extrapolations can be obtained. Certainly, the lattice results shown here indicate that improving the control of the chiral extrapolation would definitely benefit from lower masses. In fact, it seems that this is the only way one can improve the precision on the $g_{A}$ calculation both from the point of view of statistical and systematic errors. LHPC is currently working on pushing closer to the chiral limit. Using the Kogut-Susskind lattices produced by MILC they expect to be able to perform calculations in the range of $250 \mathrm{MeV}$ pions. In addition, calculations on a smaller lattice spacing are in the pipeline, in order to control the continuum extrapolation error.

In Fig. 2 we also plot recent results by RBC [74, 20] and QCDSF [71] and together with the old LHPC/SESAM results [75] obtained with two dynamical flavors of Wilson fermions. The consistency between different formulations is reassuring.

\section{Moments of structure functions}

As noted above the moments of the structure functions are related to forward matrix elements of the nucleon. In order to fix our notation for these matrix elements are related to moments as 
following

$$
\begin{aligned}
\frac{1}{2} \sum_{s}\left\langle p, s\left|\mathscr{O}_{\left\{\mu_{1} \mu_{2} \cdots \mu_{n}\right\}}^{q}\right| p, s\right\rangle & =2\left\langle x^{n-1}\right\rangle_{q}(\mu) \times\left[p_{\mu_{1}} p_{\mu_{2}} \cdots p_{\mu_{n}}+\cdots-t r\right] \\
-\left\langle p, s\left|\mathscr{O}_{\left\{\sigma \mu_{1} \mu_{2} \cdots \mu_{n}\right\}}^{5 q}\right| p, s\right\rangle & =\frac{1}{n+1} 2\left\langle x^{n}\right\rangle_{\Delta q}(\mu) \times\left[s_{\sigma} p_{\mu_{1}} p_{\mu_{2}} \cdots p_{\mu_{n}}+\cdots-t r\right] \\
\left\langle p, s\left|\mathscr{O}_{\left[\sigma\left\{\mu_{1}\right] \mu_{2} \cdots \mu_{n}\right\}}^{[5] q}\right| p, s\right\rangle & =\frac{1}{n+1} d_{n}^{q}(\mu) \times\left[\left(s_{\sigma} p_{\mu_{1}}-s_{\mu_{1}} p_{\sigma}\right) p_{\mu_{2}} \cdots p_{\mu_{n}}+\cdots-t r\right]
\end{aligned}
$$

where \{\} implies symmetrization and [] anti-symmetrization of indices. The nucleon states $|p, s\rangle$ are normalized so that $\left\langle p, s \mid p^{\prime}, s^{\prime}\right\rangle=(2 \pi)^{3} 2 E(p) \delta\left(p-p^{\prime}\right) \delta_{s, s^{\prime}}$. The operators $\mathscr{O}$ are these defined in Eq. 1.14

In addition the transversity moments are defined by

$$
\left\langle p, s\left|\mathscr{O}_{\rho v\left\{\mu_{1} \mu_{2} \cdots \mu_{n}\right\}}^{\sigma 5 q}\right| p, s\right\rangle=\frac{2}{m_{N}}\left\langle x^{n}\right\rangle_{\delta q}(\mu) \times\left[\left(s_{\rho} p_{v}-s_{v} p_{\rho}\right) p_{\mu_{1}} p_{\mu_{2}} \cdots p_{\mu_{n}}+\cdots-t r\right] .
$$

The above operators give us the moments of structure functions for arbitrary flavor. In practice however, the lattice correlation functions needed require the calculation of the so called "disconnected" diagrams. This is numerically a very challenging exercise, and hence people usually concentrate on computing the iso-vector moments for which the disconnected diagrams do not contribute. In this conference, we shaw some results of an attempt to compute these disconnected diagrams in the case of the strange quark [76].

The calculation of moments of structure functions has also been a big puzzle for lattice QCD. Traditional lattice calculations in the quenched approximation, as well as in full QCD with two light quark flavors, have been unable to reproduce the experimental results for the first few moments of both the polarized and the unpolarized moments. Published lattice results from LHPC can be found in [75] and from QCDSF in [77, 78, 66, 79, 67]. The common feature of all these calculations is the fact that relatively heavy quark masses have been used. QCDSF has performed the continuum extrapolation in the quenched approximation without any substantial change in the overall picture. $\mathrm{RBC}$ has also attempted to push to lighter pion masses using domain wall fermions in the quenched approximation $[16,17]$. They also observed no curvature that would reconcile the lattice results with the experimental expectations down to $400 \mathrm{MeV}$ pions.

It has been argued [80] that the apparent disagreement of lattice results with the experimental expectations is due to the fact that the there is a very strong chiral logarithm governing the mass dependence of these matrix elements close to the chiral limit. Thus resolving this puzzle requires computations closer to the chiral limit. QCDSF using improved Wilson fermion algorithms is currently computing these matrix elements with pion masses in the vicinity of $300 \mathrm{MeV}$.

Although the RBC quenched domain wall fermion calculations failed to observe the curvature that might reconcile the lattice with the experimental results, certain interesting features of the calculation were observed [17]. First the calculation of the twist 3 matrix elements $d_{n}$ related to the polarized structure function $g_{2}$ seems to significantly simplify. The operator $\mathscr{O}_{\left[\sigma\left\{\mu_{1}\right] \mu_{2} \cdots \mu_{n}\right\}}^{5 q}$ mixes with the operator $\mathscr{O}_{\sigma v\left\{\mu_{1} \mu_{2} \cdots \mu_{n-1}\right\}}^{\sigma q}$ if chiral symmetry is not a good symmetry, as is the case for Wilson fermions (and clover Wilson). This mixing introduces a linear divergence that needs nonperturbative subtraction in order to extract the physical matrix elements. QCDSF has successfully 

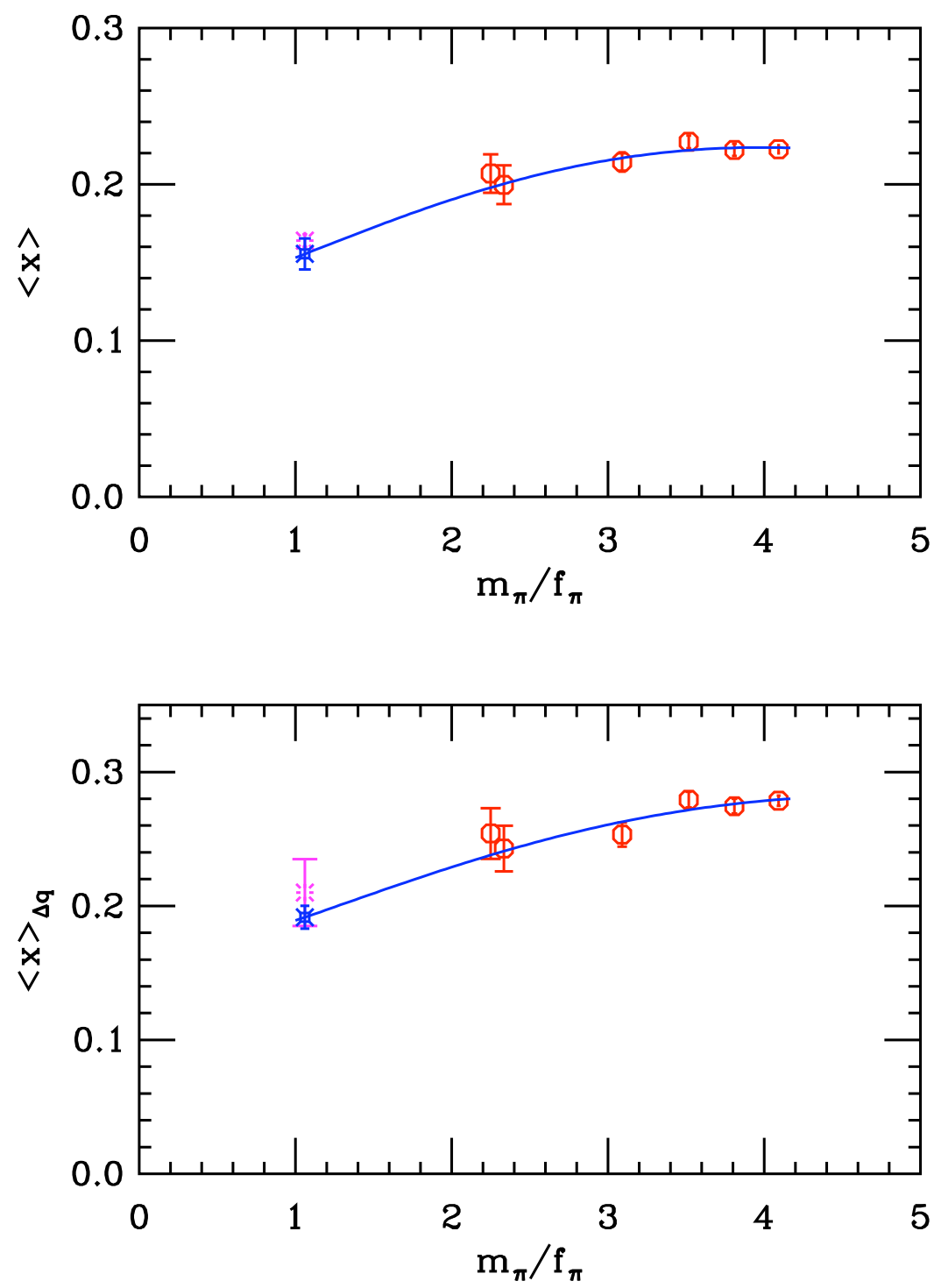

Figure 4: Chiral extrapolation of the first moments of structure functions in $\overline{M S} \mu=2 \mathrm{GeV}$. Perturbative renormalization is used.

performed this subtraction [79] for the case of the matrix element $d_{2}$. The RBC results for $d_{1}$, which has similar mixing issues to $d_{2}$, demonstrate that the domain wall fermions eliminate the need for non-perturbative subtraction. Hence, the calculation of these matrix elements significantly simplifies with the use of fermions respecting chiral symmetry on the lattice.

Furthermore, due to chiral symmetry, the renormalization constants needed for the operators $\mathscr{O}_{\left\{\sigma \mu_{1} \mu_{2} \cdots \mu_{n-1}\right\}}^{5 q}$ are the same as these of the operators $\mathscr{O}_{\left\{\mu_{1} \mu_{2} \cdots \mu_{n}\right\}}^{q}$. Hence, one can form the ratio of the lattice first moment of the quark density to the lattice first moment of the helicity distribution and obtain the ratio of the renormalized quantities, allowing direct comparison to experiment. In [17] the renormalization constants of these first moments were computed non-perturbatively with the Rome-Southampton method and found to be equal. It was also found that this ratio (in 


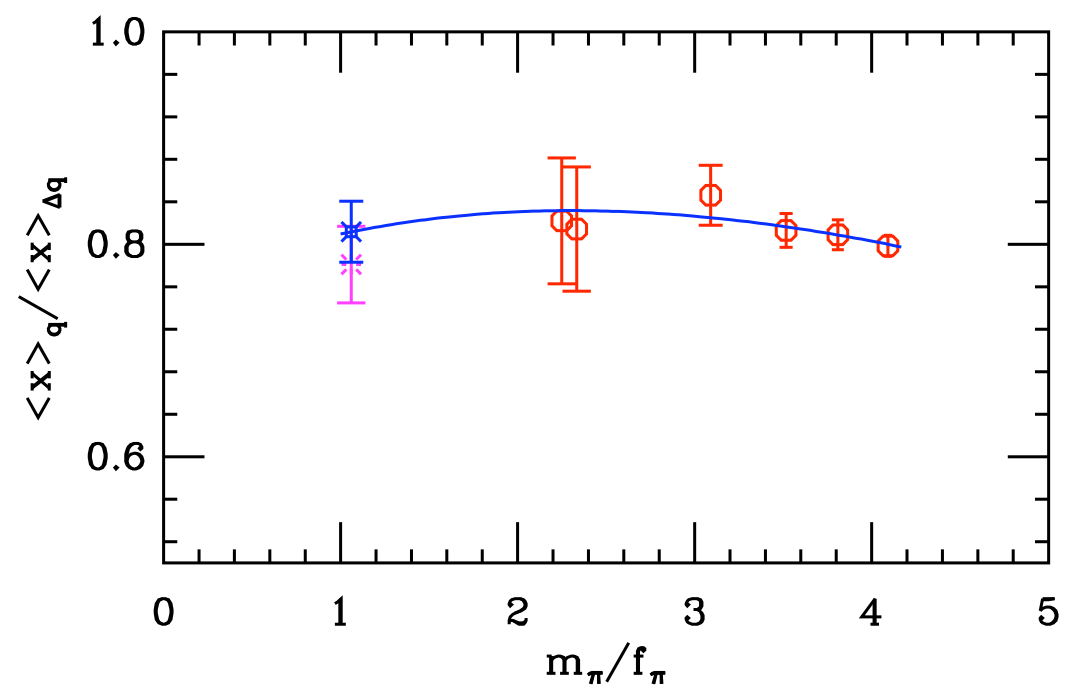

Figure 5: Chiral extrapolation of the ratio of the first moments of structure functions.

the quenched approximation) is in good agreement with the experimental ratio. The main reason for this agreement is the fact that the ratio has a smoother chiral behavior since the chiral logs partly cancel. In addition, the ratio has less systematic contamination due to uncertainties in the renormalization constant determination.

QCDSF has pushed the quenched calculations with overlap fermions to even lighter pion masses and also at significantly higher statistics. Their preliminary results ploted in Fig. 3 show curvature appearing below $400 \mathrm{MeV}$ in the first moment of the quark density distribution ${ }^{2}$

Recently [29] LHPC has completed a calculation at one lattice spacing ( $a=0.125 \mathrm{fm})$ using domain wall fermions on 2+1 Kogut-Susskind sea quarks generated by the MILC collaboration. The use of domain wall fermions in the valence sector imposes the chiral symmetry constraints that are responsible for the nice features outlined above. In addition, fitting to one loop continuum chiral perturbation theory becomes significantly simpler as calculations by NPLQCD first showed in the calculation of $\pi-\pi$ scattering lengths [30]. Subsequently, analytic results [37, 81] and applications to other observables [33, 35, 31, 32] showed similar behavior. LHPC performed the fits for all moments computed using leading order chiral perturbation theory [82, 83, 84] formulas. For example I present here the first moment of the quark density and the helicity distributions

$$
\begin{aligned}
\langle x\rangle_{u-d} & =C\left[1-2 \frac{3 g_{A}^{2}+1}{\left(4 \pi f_{\pi}\right)^{2}} m_{\pi}^{2} \ln \left(\frac{m_{\pi}^{2}}{\left(4 \pi f_{\pi}\right)^{2}}\right)+e\left(4 \pi f_{\pi}\right) \frac{m_{\pi}^{2}}{\left(4 \pi f_{\pi}\right)^{2}}\right] \\
\langle x\rangle_{\Delta u-\Delta d} & =\tilde{C}\left[1-2 \frac{2 g_{A}^{2}+1}{\left(4 \pi f_{\pi}\right)^{2}} m_{\pi}^{2} \ln \left(\frac{m_{\pi}^{2}}{\left(4 \pi f_{\pi}\right)^{2}}\right)+\tilde{e}\left(4 \pi f_{\pi}\right) \frac{m_{\pi}^{2}}{\left(4 \pi f_{\pi}\right)^{2}}\right]
\end{aligned}
$$

where the normalization is set such that the physical $f_{\pi}$ is expected to be around $132 \mathrm{MeV}$. In the above formula we have taken the scale $\mu=4 \pi f_{\pi}$. Furthermore, in the above formulas one can eliminate the unknowns of $g_{A}$ and $f_{\pi}$ at the chiral limit by replacing them with their values measured on the lattice at a given pion mass. This amounts to a modification of the above formula

\footnotetext{
${ }^{2}$ Figure contributed by G. Schierholz.
} 


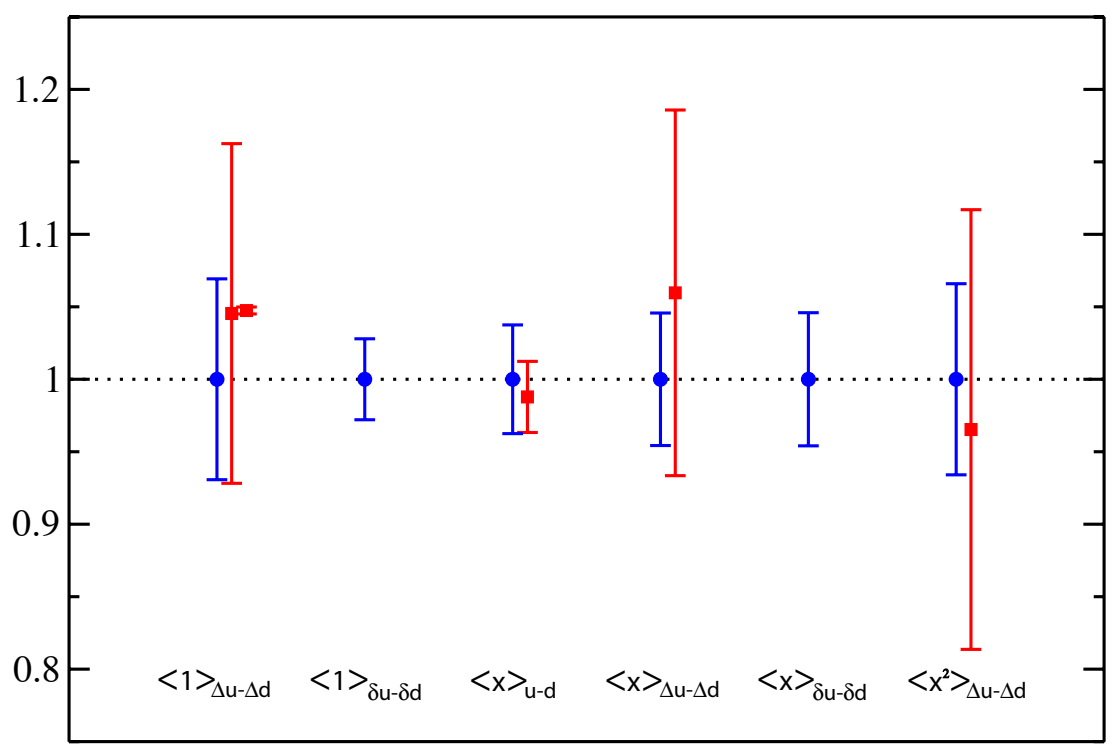

Figure 6: The ratio of experiment vs lattice (red points) for the iso-vector moments. The small errorbar for $\langle 1\rangle_{D u-D d}$ comes from neutron beta decay experiments, while the rest come from DIS experiments. The blue points denote the lattice statistical error bar, while the red error bars are the experimental errors on these quantities.

at higher order. If we do this we have only two unknown fit parameters: the counter term and the value of the matrix element at the chiral limit. In Fig. 4 the fits done with the above method are presented. We see that there is a hint of a curvature for the point at $350 \mathrm{MeV}$ (lightest point).

The extrapolated results obtained by evaluating Eq. 5.4 at the physical $m_{\pi} / f_{\pi}$ point using the fitted values for the counter terms and the parameters $C$ and $\tilde{C}$ are in remarkable agreement with the experimental expectations. The results of such fits where presented by Renner [29] in this conference. A summary of his results is shown in Fig. 6. As we can see all lattice extrapolated results are in a very good agreement with experiment and in all cases, the deep inelastic scattering experimental error is larger than the lattice statistical error. Further investigation of these fits is required in order to assess possible systematic errors. One certainly needs to perform the continuum extrapolation to remove all discretization errors. In addition, the LHPC results are perturbatively renormalized and hence, non-perturbative renormalization is needed to eliminate the remaining renormalization systematic error. However, one can look at particular ratios of the unrenormalized matrix elements, which require no further normalization factors due to the domain wall fermion chiral symmetry. This ratio for the first moments of the quark density and helicity distribution is plotted in Fig. 5 and indeed is in agreement with experiment at the physical point. 


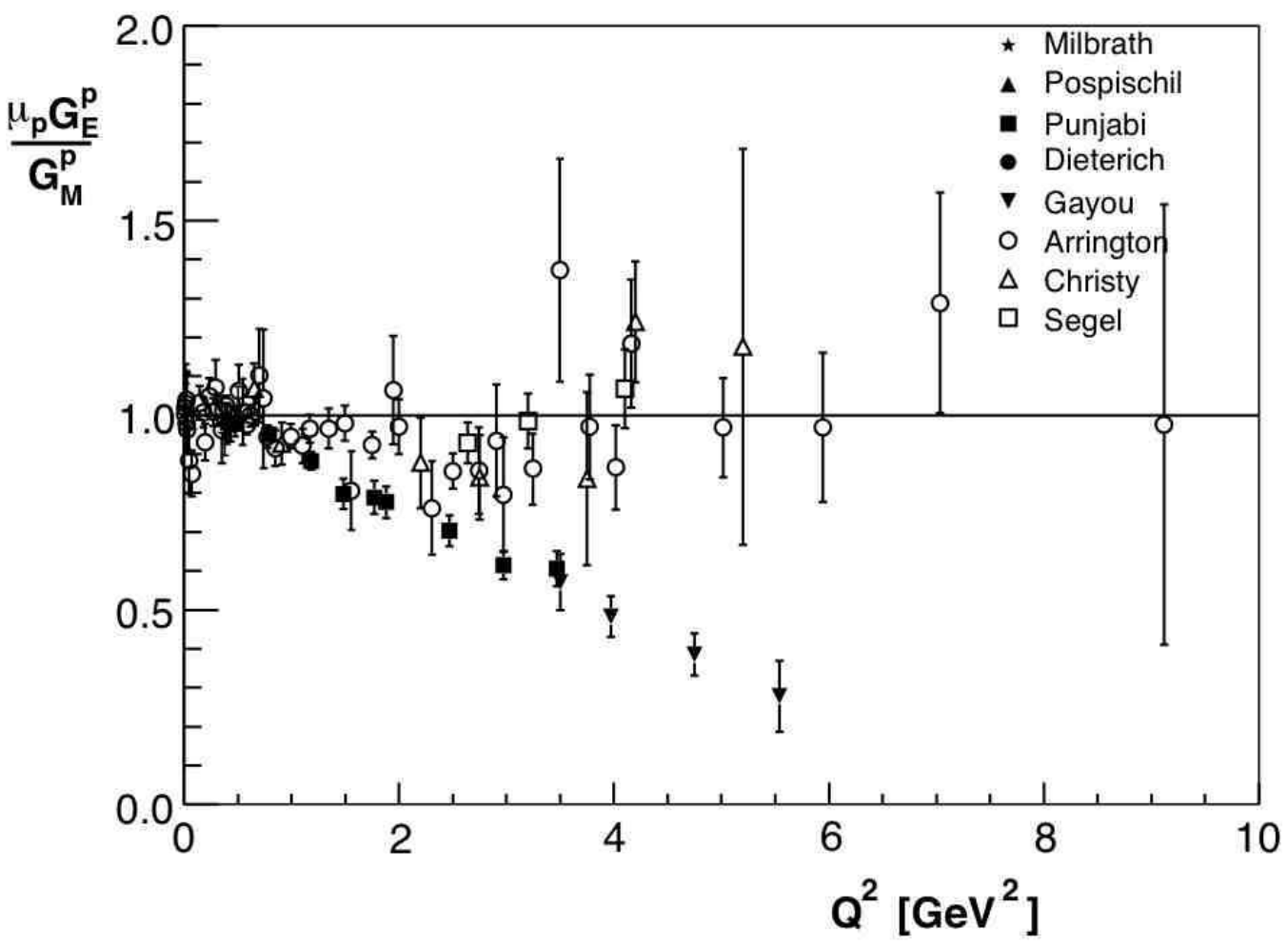

Figure 7: Experimental determinations of the ratio $G_{E} / G_{M}$ for the proton [85]. The open symbols correspond to the Rosenbluth determinations of $G_{E} / G_{M}$ [86, 87, 88, 89]. The solid symbols come from polarization data experiments at JLab [90, 91, 92].

\section{Form Factors}

In the last few years a lot of interesting experimental results for nucleon elastic form factors have appeared. The measurements at Jlab using polarization transfer [93, 90, 91, 92] for extracting the form factors, and extending the $Q^{2}$ region to $6 \mathrm{GeV}^{2}$ contradicted results based on the Rosenbluth separation technique [87, 88, 89]. Hence the old belief that the ratio of the electric to magnetic form factor is one if scaled by the magnetic moment was questioned. For a compilation of the experimental results see Fig. 7. After careful examination of the two techniques it was realized that the polarization transfer data are less prone to systematic errors due to two photon effects $[94,95,96]$; hence the true behavior of the ratio is the one measured in polarization transfer experiments.

These experimental results renewed the interest in lattice calculations of nucleon form factors. There are three recent calculations available. The LHPC calculation [29] which is based on the 2+1 flavor dynamical mixed action calculation scheme, the QCDSF calculation [97] with 2 dynamical flavors of improved Wilson fermions (and improved currents), and the unimproved Wilson fermion (quenched and 2 flavor dynamical) calculation by Alexandrou et.al. [98].

In Fig. 8 the LHPC results for the iso-vector $F_{1}$ form factor are ploted together with the ex- 


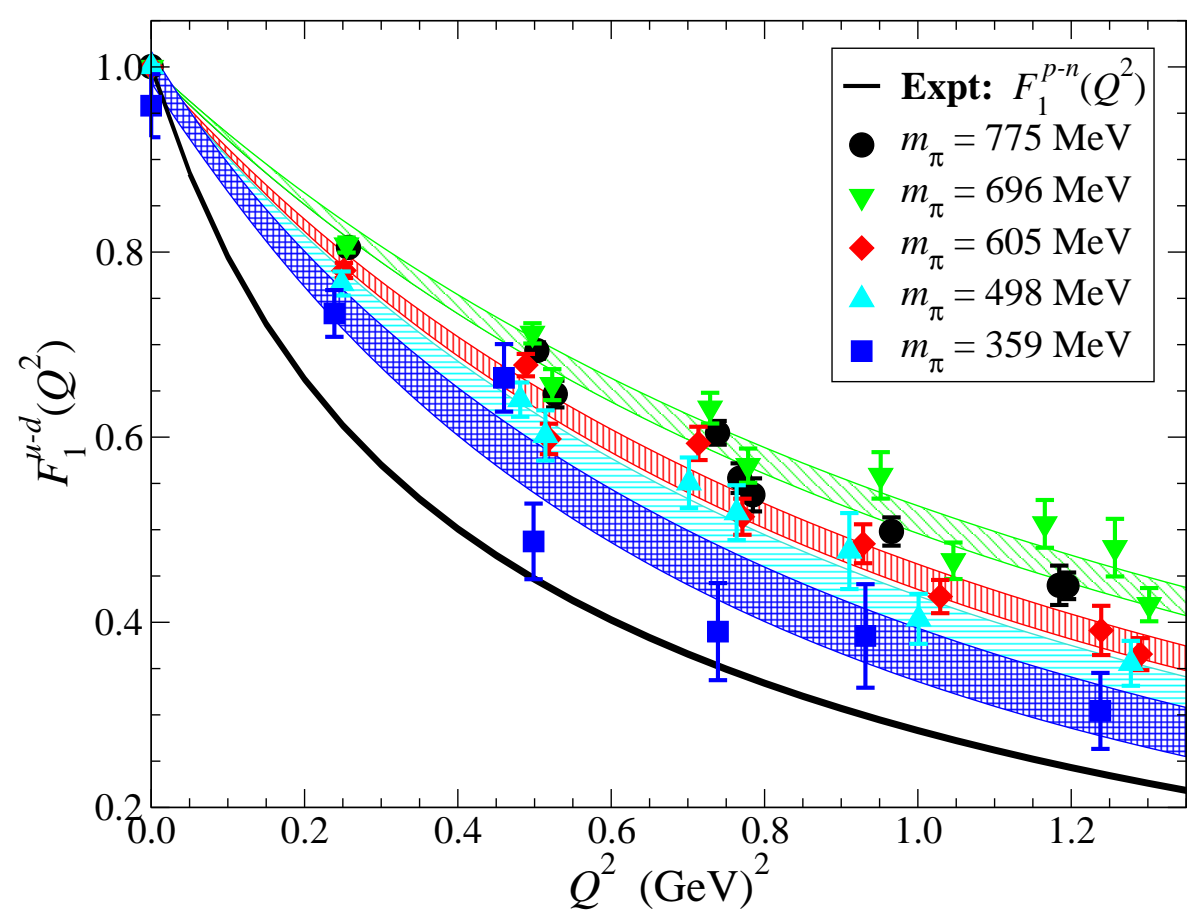

Figure 8: $F_{1}^{u-d}\left(Q^{2}\right)$ for several pion masses. The black band represents the experimental error based on data by [99].

perimental error band ${ }^{3}$. We can see that as the pion mass approaches the physical point the lattice data are approaching experiment. LHPC performed a fit using the chiral perturbation formulas of [100] to the rms radius and found that the extrapolated result is close to the experimental. A hint of the expected curvature in the rms radius extrapolation is evident in the data. In addition LHPC presented preliminary results on the axial form factors and the high $Q^{2}$ region which is expected to be of high interest due to upcoming experiments.

QCDSF presented their results in this conference as well [97]. These new results are extending to lighter pion masses the results presented earlier in [101], where it was clear that in order to make contact to chiral perturbation theory one needed lighter pion masses. Here, they focus on determining the appropriate fit formulas for the form factors as a function of $Q^{2}$. They conclude that the statistical precision is not sufficient for a firm conclusion. However, they observed differences in the $Q^{2}$ dependence of $F_{1}$ and $F_{2}$ and between the contributions of the up and down flavors, as Fig. 9 indicates ${ }^{4}$. They also studied possible chiral perturbation fits to the rms radii identifying possible strong effects as one approaches the physical point, in accordance with the expectations of chiral perturbation theory.

Finally results from the calculation by Alexandrou et al. are ploted in Fig. 10. The figure is taken from [98]. Evidently, the lattice results seem to disagree with the experimental numbers

\footnotetext{
${ }^{3}$ I thank G. Fleming for computing the experimental error band and providing this figure. The errorband was based on data by [99].

${ }^{4}$ I thank D. Pleiter for providing this figure.
} 


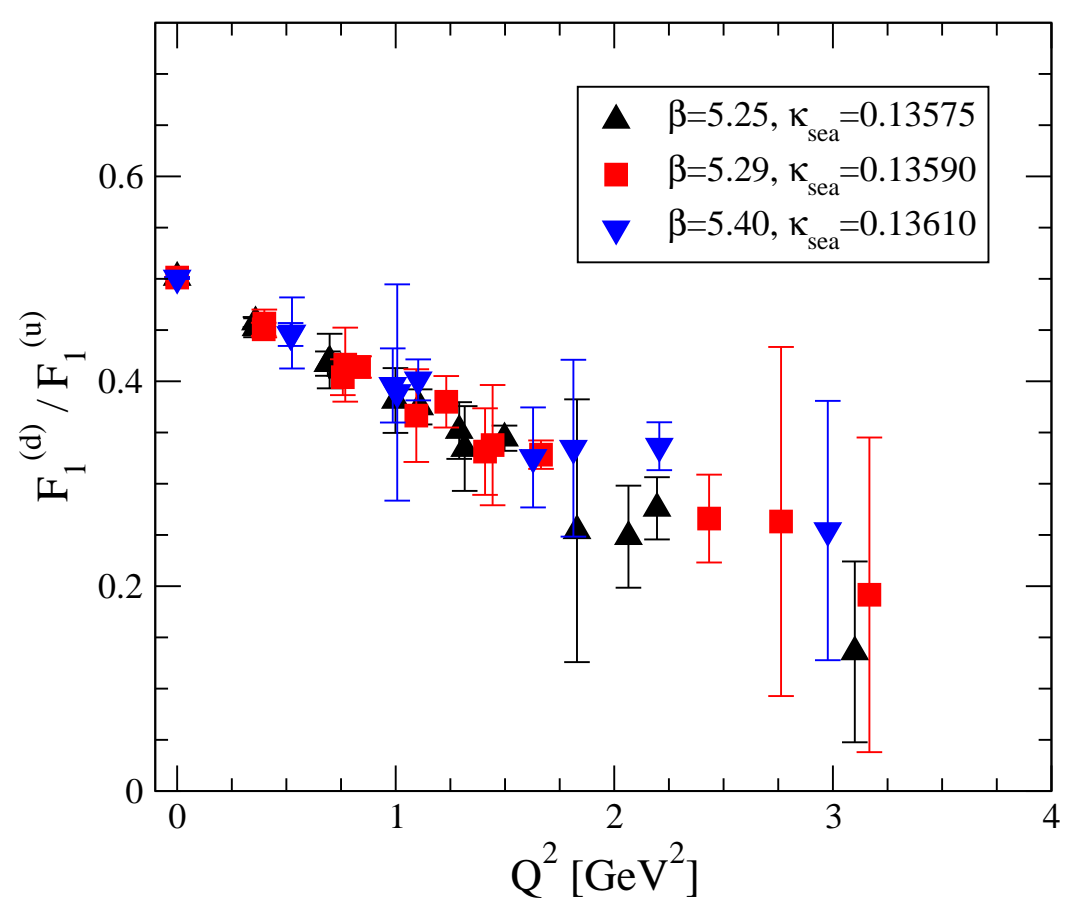

Figure 9: The ratio of up and down contributions to $F_{1}$ (connected only) for $m_{\pi}=400 \mathrm{MeV}$ [97].

throughout the range of the plotted $Q^{2}$ dependence. These results are quenched except two points which are two flavor dynamical Wilson fermion calculations. The deviation might be due to lattice spacing errors which one expects to become larger as $Q^{2}$ grows. In addition, Alexandrou et al. presented their recent results on the Nucleon to Delta transition form factors [102, 103].

One needs to carefully study the results from different calculations in order to understand how various systematic errors affect the lattice calculations of form factors. Given the different calculations available better understanding of these effects is expected in the near future.

\subsection{Strangeness in the nucleon}

One important experimental goal is to understand the strange quark contributions to the elctromagnetic form factors of the nucleon. The most recent experiments by HAPPEX [104, 105] and G0 [106] at JLab, together with the older ones by SAMPLE at MIT-BATES [107], and by A4 at Mainz $[108,109]$, confirm that there is a non-zero strange contribution to the proton elctromagnetic form factors. However, the size of these contributions is rather small.

Although lattice calculations of disconnected diagrams such as these contributing to the strange form factors are rather difficult, there are already two lattice calculations of the strange electric and magnetic form factors of the proton. Fist Lewis et al. [110] used Wilson fermions in the quenched approximation and performed the needed chiral extrapolations in the context of quenched chiral perturbation theory. Second Leinweber et al. [111] used FLIC fermions again in the quenched approximation. They employed a combination of lattice QCD, charge symmetry and chiral perturbattion theory and avoid direct computation of the disconnected diagrams. Both calculations produced results consistent with current experimental data. 


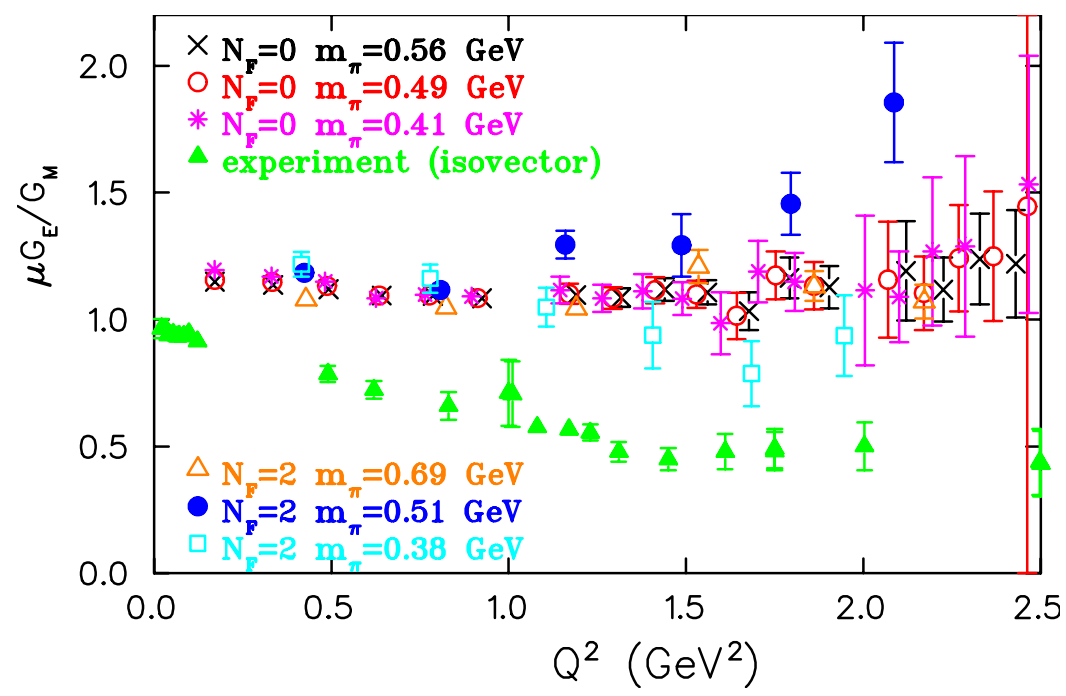

Figure 10: The ratio of the electric to magnetic iso vector form factors by Alexandrou et al. [98].

\subsection{Neutron electric dipole moment}

Over the last couple of years, calculations of the neutron electric dipole moment $\left(d_{N}\right)$ due to the existence of a non-trivial theta term in the QCD action were also revived. When such a term exists, time reversal and parity symmetries are broken. Hence, CP violating terms such as the electric dipole moment, appear in the electromagnetic form factors of the nucleon. Experimentally no permanent EDM of fundamental particles has been observed, requiring that the coefficient $(\theta)$ with which the theta term enters the QCD Lagrangian must vanish. This is known as the strong CP problem. In other words $\theta$ seems to be fine tuned to zero. The most stringent experimental limit on the existence of an EDM comes from the neutron where $d_{N}<6.3 \times 10^{-26} \mathrm{ecm}$. This in turn implies the most stringent experimental constrain on $\theta$. The relation between the neutron EDM and $\theta$ can only be reliably estimated non-perturbatively. Hence lattice QCD calculations are needed.

There are two ways one can compute the electric dipole moment (EDM). The first relies on computations of the regular vector electromagnetic form factor isolating the CP violating term proportional to the EDM. The other is using the background field method to extract the EDM by measuring the mass splitting of the neutron with spin aligned or anti-aligned with the external field. In both cases, one uses re-weighting in order to include the effects of the non-positive definite theta term. Since the EDM is experimentally small re-weighting should work well.

CP-PACS performed a quenched calculation with domain wall fermions [112]. However, the quenched calculation for this quantity is very misleading since the electric dipole moment is expected to diverge in the quenched chiral limit due to the fact that the topological susceptiblility remains finite in this limit. In this conference CP-PACS presented their new results in quenched and dynamical calculations using the external field method [113]. In both cases they were able to obtain a non-vanishing signal.

Berruto et al. $[114,115]$ performed the first two flavor dynamical domain wall fermion calculations using the form factor method with re-weighting. Their result, together with the experimental 
bound [116] on the neutron EDM, yields $\theta<6.3 \times 10^{-11}$.

Finally, in this conference QCDSF presented their first results using high statistics quenched overlap fermions [117].

\subsection{Polarizabilities}

The polarizabilities of the nucleon describe its response to external electric or magnetic fields. They are the coefficients appearing in the low momentum transfer expansion of the hadronic tensor in Compton scattering, and carry important information about nucleon structure. The recent relation of two photon processes to the systematic errors appearing in the experimental extraction of the electromagnetic form factors of the nucleon via the Rosenbluth separation technique, has renewed the interest in understanding Compton scattering. The polarizabilities describe the low energy limit of this process.

The simplest method of performing these calculations in lattice QCD is the background field method. Lattice results in the quenched approximation exist for both the electric $[118,119]$ and the magnetic polarizabilities [120].

Recently [121, 122], it was shown how the background field method can be used to extract the spin polarizabilities. In the same publication, the finite volume corrections were computed in chiral perturbation theory indicating a surprisingly large dependence on both the volume and the quark mass $(O(10 \%))$.

\section{Moments of generalized parton distribution functions}

As mentioned in the introduction, on the lattice we can compute nucleon matrix elements of the twist 2 operators. These matrix elements are generalized form factors that are the coefficients of the polynomial expansion in $\xi$ of the moments of the generalized parton distribution functions. Such calculations are a straight forward extension to the off forward limit of the standard lattice calculations of the moments of the parton distribution functions. The relation of matrix elements and generalized form factors is given by (see for example [124])

$$
\begin{aligned}
& \left\langle p^{\prime}\left|\mathscr{O}_{\mu_{1}}^{q}\right| p\right\rangle=\left\langle\left\langle\gamma_{\mu_{1}}\right\rangle\right\rangle A_{10}(t)+\frac{\mathrm{i}}{2 m}\left\langle\left\langle\sigma_{\mu_{1} \alpha}\right\rangle\right\rangle \Delta_{\alpha} B_{10}(t), \\
& \left\langle p^{\prime}\left|\mathscr{O}_{\left\{\mu_{1} \mu_{2}\right\}}^{q}\right| p\right\rangle=\bar{p}_{\left\{\mu_{1}\right.}\left\langle\left\langle\gamma_{\left.\mu_{2}\right\}}\right\rangle\right\rangle A_{20}(t)+\frac{\mathrm{i}}{2 m} \bar{p}_{\left\{\mu_{1}\right.}\left\langle\left\langle\sigma_{\left.\mu_{2}\right\} \alpha}\right\rangle\right\rangle \Delta_{\alpha} B_{20}(t)+\frac{1}{m} \Delta_{\left\{\mu_{1}\right.} \Delta_{\left.\mu_{2}\right\}} C_{20}(t), \\
& \left\langle p^{\prime}\left|\mathscr{O}_{\left\{\mu_{1} \mu_{2} \mu_{3}\right\}}^{q}\right| p\right\rangle=\bar{p}_{\left\{\mu_{1}\right.} \bar{p}_{\mu_{2}}\left\langle\left\langle\gamma_{\left.\mu_{3}\right\}}\right\rangle\right\rangle A_{30}(t)+\frac{\mathrm{i}}{2 m} \bar{p}_{\left\{\mu_{1}\right.} \bar{p}_{\mu_{2}}\left\langle\left\langle\sigma_{\left.\mu_{3}\right\} \alpha}\right\rangle\right\rangle \Delta_{\alpha} B_{30}(t) \\
& +\Delta_{\left\{\mu_{1}\right.} \Delta_{\mu_{2}}\left\langle\left\langle\gamma_{\left.\mu_{3}\right\}}\right\rangle\right\rangle A_{32}(t)+\frac{\mathrm{i}}{2 m} \Delta_{\left\{\mu_{1}\right.} \Delta_{\mu_{2}}\left\langle\left\langle\sigma_{\left.\mu_{3}\right\} \alpha}\right\rangle\right\rangle \Delta_{\alpha} B_{32}(t),
\end{aligned}
$$

for the unpolarized case,

$$
\begin{aligned}
\left\langle p^{\prime}\left|\mathscr{O}_{\mu_{1}}^{5 q}\right| p\right\rangle & =\left\langle\left\langle\gamma_{\mu_{1}} \gamma_{5}\right\rangle\right\rangle \widetilde{A}_{10}(t)+\frac{1}{2 m} \Delta_{\mu_{1}}\left\langle\left\langle\gamma_{5}\right\rangle\right\rangle \widetilde{B}_{10}(t), \\
\left\langle p^{\prime}\left|\mathscr{O}_{\left\{\mu_{1} \mu_{2}\right\}}^{5 q}\right| p\right\rangle & =\bar{p}_{\left\{\mu_{1}\right.}\left\langle\left\langle\gamma_{\left.\mu_{2}\right\}} \gamma_{5}\right\rangle\right\rangle \widetilde{A}_{20}(t)+\frac{1}{2 m} \Delta_{\left\{\mu_{1}\right.} \bar{p}_{\left.\mu_{2}\right\}}\left\langle\left\langle\gamma_{5}\right\rangle\right\rangle \widetilde{B}_{20}(t),
\end{aligned}
$$



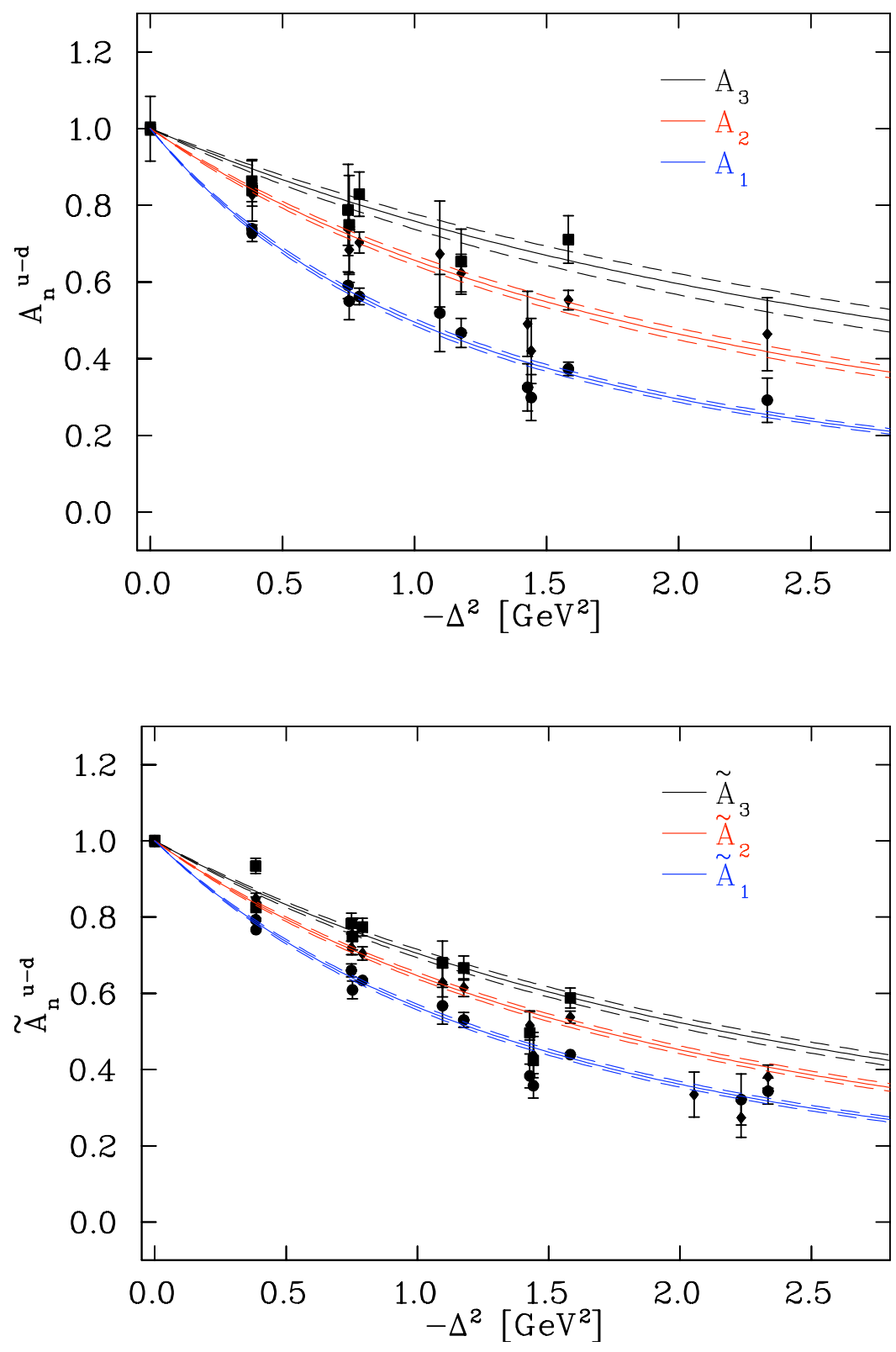

Figure 11: The generalized form factors from QCDSF [123].

$$
\begin{aligned}
\left\langle p^{\prime}\left|\mathscr{O}_{\left\{\mu_{1} \mu_{2} \mu_{3}\right\}}^{5 q}\right| p\right\rangle & =\bar{p}_{\left\{\mu_{1}\right.} \bar{p}_{\mu_{2}}\left\langle\left\langle\gamma_{\left.\mu_{3}\right\}} \gamma_{5}\right\rangle\right\rangle \widetilde{A}_{30}(t)+\frac{1}{2 m} \Delta_{\left\{\mu_{1}\right.} \bar{p}_{\mu_{2}} \bar{p}_{\left.\mu_{3}\right\}}\left\langle\left\langle\gamma_{5}\right\rangle\right\rangle \widetilde{B}_{30}(t) \\
& +\Delta_{\left\{\mu_{1}\right.} \Delta_{\mu_{2}}\left\langle\left\langle\gamma_{\left.\mu_{3}\right\}} \gamma_{5}\right\rangle\right\rangle \widetilde{A}_{32}(t)+\frac{1}{2 m} \Delta_{\left\{\mu_{1}\right.} \Delta_{\mu_{2}} \Delta_{\left.\mu_{3}\right\}}\left\langle\left\langle\gamma_{5}\right\rangle\right\rangle \widetilde{B}_{32}(t),
\end{aligned}
$$

for the polarized, and

$$
\left\langle p^{\prime}\left|\mathscr{O}_{\mu v}^{\sigma q}\right| p\right\rangle=\left\langle\left\langle\sigma_{\mu v}\right\rangle\right\rangle A_{T 10}(t)-\frac{\mathrm{i}}{m^{2}}\langle\langle 1\rangle\rangle \bar{p}_{[\mu} \Delta_{v]} \widetilde{A}_{T 10}(t)-\frac{\mathrm{i}}{2 m}\left\langle\left\langle\gamma_{[\mu}\right\rangle\right\rangle \Delta_{v]} B_{T 10}(t),
$$




$$
\begin{aligned}
\left\langle p^{\prime}\left|\mathscr{O}_{\mu v \mu \mu_{1}}^{\sigma q}\right| p\right\rangle & =\left\langle\left\langle\sigma_{\mu\{v}\right\rangle\right\rangle \bar{p}_{\left.\mu_{1}\right\}} A_{20}(t)-\frac{\mathrm{i}}{m^{2}}\langle\langle 1\rangle\rangle \bar{p}_{[\mu} \Delta_{\{v]} \bar{p}_{\left.\mu_{1}\right\}} \widetilde{A}_{T 20}(t) \\
& -\frac{\mathrm{i}}{2 m}\left\langle\left\langle\gamma_{[\mu}\right\rangle\right\rangle \Delta_{\{v]} \bar{p}_{\left.\mu_{1}\right\}} B_{T 20}(t)-\frac{\mathrm{i}}{m}\left\langle\left\langle\gamma_{[\mu}\right\rangle\right\rangle \bar{p}_{\{v]} \Delta_{\left.\mu_{1}\right\}} B_{T 21}(t), \\
\left\langle p^{\prime}\left|\mathscr{O}_{\mu v \mu_{1} \mu_{2}}^{\sigma q}\right| p\right\rangle & =\left\langle\left\langle\sigma_{\mu\{v}\right\rangle\right\rangle \bar{p}_{\mu_{1}} \bar{p}_{\left.\mu_{2}\right\}} A_{30}(t)+\left\langle\left\langle\sigma_{\mu\{v}\right\rangle\right\rangle \bar{\Delta}_{\mu_{1}} \Delta_{\left.\mu_{2}\right\}} A_{32}(t), \\
& -\frac{\mathrm{i}}{m^{2}}\langle\langle 1\rangle\rangle \bar{p}_{[\mu} \Delta_{\{v]} \bar{p}_{\mu_{1}} \bar{p}_{\left.\mu_{2}\right\}} \widetilde{A}_{T 30}(t)-\frac{\mathrm{i}}{m^{2}}\langle\langle 1\rangle\rangle \bar{p}_{[\mu} \Delta_{\{v]} \Delta_{\mu_{1}} \Delta_{\left.\mu_{2}\right\}} \widetilde{A}_{T 32}(t), \\
& -\frac{\mathrm{i}}{2 m}\left\langle\left\langle\gamma_{[\mu}\right\rangle\right\rangle \Delta_{\{v]} \bar{p}_{\mu_{1}} \bar{p}_{\left.\mu_{2}\right\}} B_{T 30}(t)-\frac{\mathrm{i}}{2 m}\left\langle\left\langle\gamma_{[\mu}\right\rangle\right\rangle \Delta_{\{v]} \Delta_{\mu_{1}} \Delta_{\left.\mu_{2}\right\}} B_{T 32}(t) \\
& -\frac{\mathrm{i}}{m}\left\langle\left\langle\gamma_{[\mu}\right\rangle\right\rangle \bar{p}_{\{v]} \Delta_{\mu_{1}} \bar{p}_{\left.\mu_{2}\right\}} B_{T 31}(t),
\end{aligned}
$$

for the transverse GPDs. In the above $t=-\Delta^{2}, \Delta=p^{\prime}-p, \bar{p}=\left(p^{\prime}+p\right) / 2,\{\}$ denotes symmetrization while [] denotes anti-symmetrization, and $\langle\langle\Gamma\rangle\rangle=\bar{u}\left(p^{\prime}, s^{\prime}\right) \Gamma u(p, s)$.

Both LHPC/SESAM [125] and QCDSF [126] almost simultaneously published their first results on the moments of GPDs using two flavor dynamical Wilson fermions. These results were obtained with relatively heavy pion masses, however, the expected qualitative behavior was already observed. In particular, as seen in Fig. 11, the generalized form factors become flatter at higher moments. This is expected since for higher moments the region closer to $x=1$ contributes more to the integral over the momentum fraction, resulting in milder momentum space dependence on the transverse momentum transfer. In other words, this observation is a direct consequence of the fact that the probability distribution becomes narrower when $x$ approaches one, as depicted in Fig. 1. Both collaborations are now pushing towards the physical pion masses. QCDSF is using two flavor improved dynamical Wilson fermions, while LHPC is using the mixed action scheme with domain wall fermions in the valence sector and 2+1 flavors of Kogut-Susskind fermions in the sea.

LHPC presented their results in this conference [29]. In Fig. 12 we show their results for the case of upolarized GPDs. In addition, in Fig. 13 the lattice data are compared to phenomenological fits to generalized parton distributions introduced by Diehl et al. [127]. It is evident that as the pion mass gets smaller the slope of the ratio $A_{30} / A_{10}$ becomes closer to phenomenological expectations.

Finally, in this conference QCSF presented their results on transverse generalized parton distribution functions [128] (see also [129, 130, 131, 132]). By computing the moments of the transverse parton distribution functions, one can perform the inverse Fourier transformation and produce a two dimensional image of the nucleon. The transverse spin densities in the impact parameter space are particularly interesting because they provide a qualitative understanding of the single spin asymmetries such as the Sivers asymmetry [133, 134] and the Boer-Mulders effect [135], observed in semi-inclusive deep inelastic scattering experiments.

\subsection{The spin of the proton}

The nucleon, being a composite object, acquires its spin from its constituents. Quantum effects, though, make it difficult to understand how spin arises. The naive expectation that the proton 


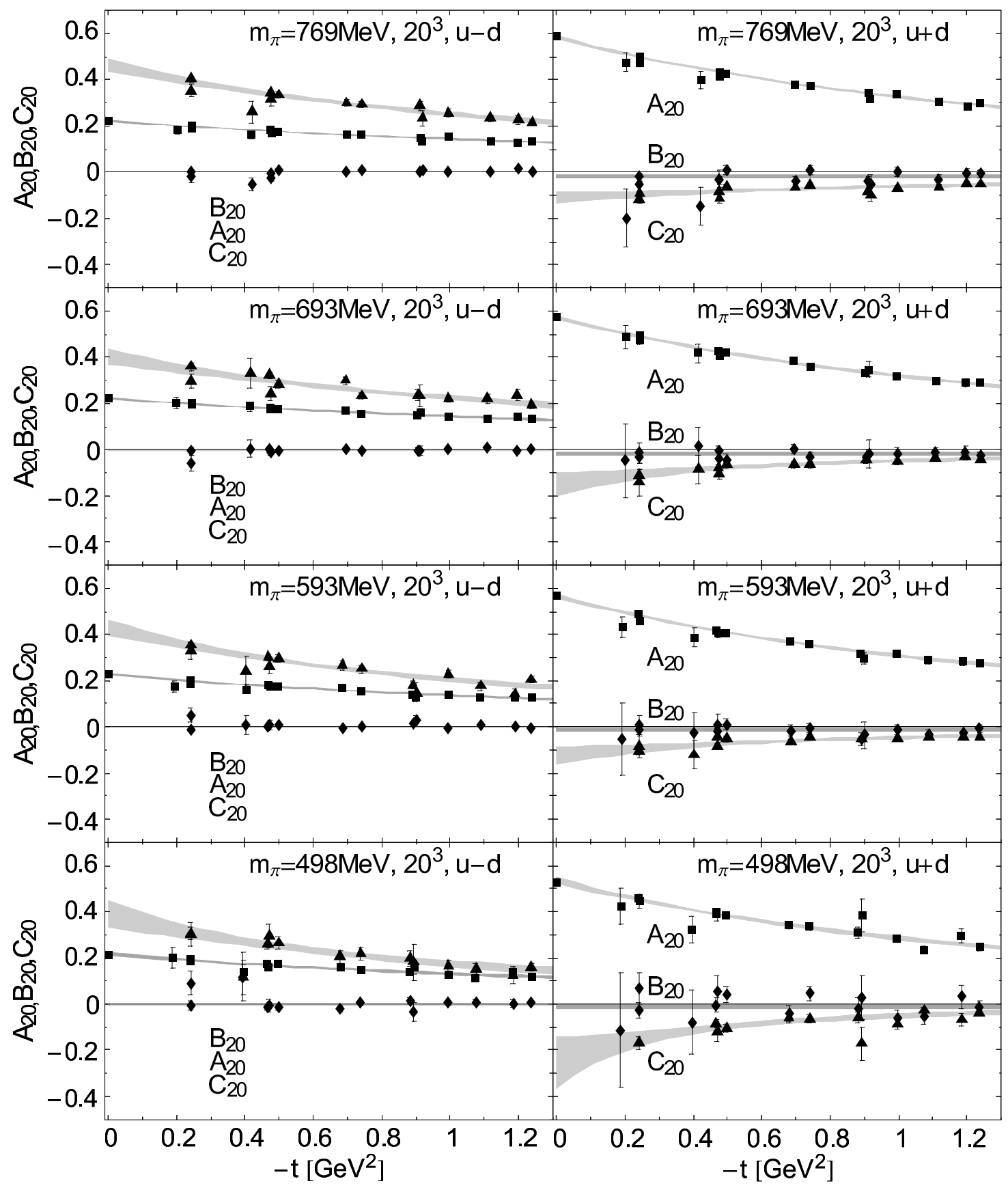

Figure 12: The generalized form factors from LHPC [29]. 


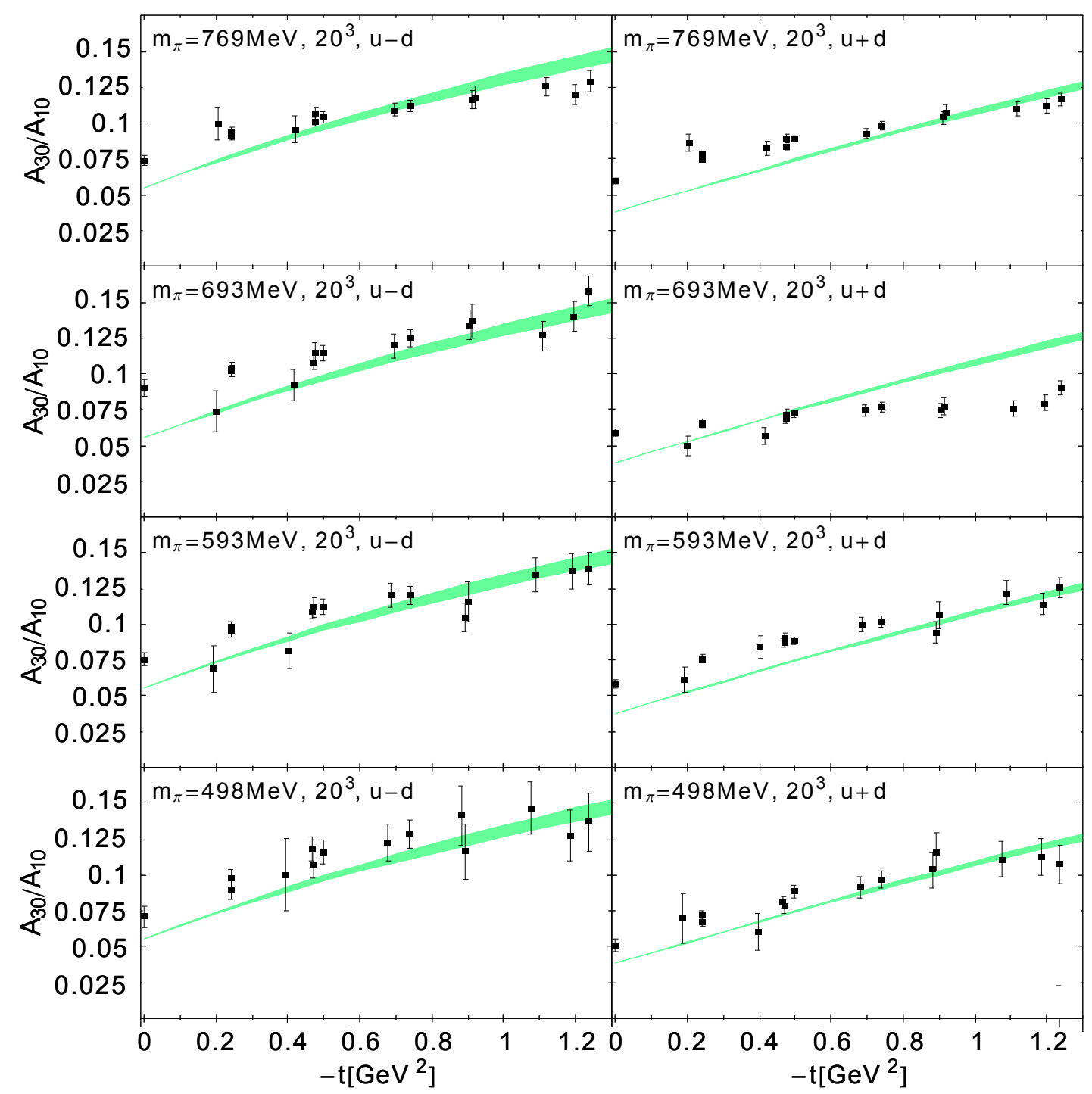

Figure 13: The generalized form factors from LHPC [29]. A comparison of lattice results to a phenomenological fit to generalized parton distribution functions [127, 29].

spin is carried by the constituent quarks has been proven wrong experimentally, giving rise to the proton spin "crisis". The proton spin can be decomposed [5, 136, 137] as follows

$$
\frac{1}{2}=\frac{1}{2} \Delta \Sigma+\Delta g+L_{z} \quad \text { or } \quad \frac{1}{2}=\frac{1}{2} \Delta \Sigma+L_{z}^{q}+J_{z}^{g},
$$

where $\Delta \Sigma=\Delta u+\Delta d+\Delta s$ is the quarks spin, $\Delta g$ is the spin of the gluons $L_{z}$ is the total orbital angular momentum of the quarks and gluons, $L_{z}^{q}$ is the quark orbital angular momentum and, $J_{z}^{g}$ is the total gluon angular momentum. Note that the decomposition of the proton spin can be done in only three terms (for example see [5, 136]) and not four as the naive quark model might imply. Experimentally $\Delta \Sigma$, which is a scale dependent quantity, is found to be almost zero within 
experimental errors, by both the EMS (at $10 \mathrm{GeV}^{2}$ ) and $\mathrm{SMC}$ (at $5 \mathrm{GeV}^{2}$ ) experiments.

From the point of view of generalized parton distribution functions it was shown [5] that the generalized form factors of the first unpolarized moment, are related to the total angular momentum of the quarks i.e.

$$
J_{z}^{q}=\frac{1}{2} \Delta \Sigma+L_{z}^{q}=\frac{1}{2}\left[A_{20}(0)+B_{20}(0)\right] .
$$

Note the the operator whose generalized form factors are $A_{20}$ and $B_{20}$ is nothing but the energy momentum tensor:

$$
\mathscr{O}_{\{\mu v\}}^{q}=\frac{i}{2} \gamma_{\{\mu} \stackrel{\leftrightarrow}{D}_{v\}}-\text { trace }=T_{\mu v}^{q}
$$

Likewise the form factors $A_{20}^{g}$ and $B_{20}^{g}$ of the energy momentum tensor for the gluons $T_{\mu \nu}^{g}$ give the total gluon angular momentum:

$$
J_{z}^{g}=\frac{1}{2}\left[A_{20}^{g}(0)+B_{20}^{g}(0)\right] .
$$

The first lattice calculation of the total angular momentum of the quarks inside the nucleon was performed by Mathur et al. in the quenched approximation with Wilson fermions. In this calculation the disconnected diagrams needed for the flavor singlet operators were computed using the stochastic $Z_{2}$ noise method.

Subsequently, LHPC and QCDSF computed the same quantities as part of their first generalized parton distribution calculations. In both cases heavy two flavor dynamical Wilson quarks were used and the disconnected diagrams were ignored. QCDSF and LHPC are currently pushing the calculations towards the chiral limit. Their results show a remarkable cancelation between the up and down quark orbital angular momentum. In addition, the total contribution from the quark spin is higher than the experimental expectations, although it seems that the chiral extrapolation of this observable may result in numbers comparable with experiment. In both calculations the disconnected diagrams have to be included for them to be complete.

\section{Conclusions}

There has been a large effort in the lattice community to compute the properties and structure of the nucleon directly from QCD. Significant effort has been devoted in understanding the sources of systematic errors and steady steps have been taken to control them. The observed agreement of results with experimental expectations enhances the confidence that the calculations are indeed controlling the systematic errors. In particular, it seems that the long standing disagreement between experiment and lattice calculations of moments of structure functions is finally resolved. Future lattice calculations will hopefully confirm this finding. Taking advantage of new theoretical developments in the field of generalized parton distributions, lattice calculations can now compute two dimensional images of the proton, helping to get further insight into the distribution of partons inside the nucleon. The synergy between lattice calculations together with experiments may soon become the way to fully utilize the power of generalized parton distributions in understanding nucleon structure. 


\section{Acknowledgments}

I would like to thank, D. Alexandrou, G. Fleming, Ph. Hägler, J. Negele, D. Pleiter, D. Renner, and G. Schierholz for helpful discussions and for contributing material (data and figures) for this manuscript. I apologize for not being able to cover other interesting topics in hadronic structure due to time constrains. This work was supported in part by DOE con- tract DE-AC05-84ER40150, under which SURA operated the Thomas Jefferson National Accelerator Facility (JLab) and in part by DOE contract DE-AC05-06OR23177 under which Jefferson Science Associates, LLC currently operates JLab.

\section{References}

[1] D. Mueller, D. Robaschik, B. Geyer, F.M. Dittes, J. Horejsi, Fortschr. Phys. 42, 101 (1994), hep-ph/9812448

[2] X.D. Ji, Phys. Rev. Lett. 78, 610 (1997), hep-ph/9603249

[3] A.V. Radyushkin, Phys. Rev. D56, 5524 (1997), hep-ph/9704207

[4] M. Diehl, T. Gousset, B. Pire, J.P. Ralston, Phys. Lett. B411, 193 (1997), hep-ph/9706344

[5] X.D. Ji, J. Phys. G24, 1181 (1998), hep-ph/9807358

[6] J. Blumlein, B. Geyer, D. Robaschik, Nucl. Phys. B560, 283 (1999), hep-ph/9903520

[7] M. Burkardt, Phys. Rev. D62, 071503 (2000), hep-ph/0005108

[8] M. Diehl, Phys. Rept. 388, 41 (2003), hep-ph/0307382

[9] A.V. Belitsky, A.V. Radyushkin, Phys. Rept. 418, 1 (2005), hep-ph/0504030

[10] J.P. Ralston, B. Pire, Phys. Rev. D66, 111501 (2002), hep-ph/0110075

[11] M. Diehl, Eur. Phys. J. C25, 223 (2002), hep-ph/0205208

[12] A.V. Belitsky, D. Mueller, Nucl. Phys. A711, 118 (2002), hep-ph/0206306

[13] M.V. Polyakov, Phys. Lett. B555, 57 (2003), hep-ph/0210165

[14] A. Freund, Eur. Phys. J. C31, 203 (2003), hep-ph/0212017

[15] M. Burkardt, Int. J. Mod. Phys. A18, 173 (2003), hep-ph/0207047

[16] K. Orginos (RBC), Nucl. Phys. Proc. Suppl. (Lattice 2002) (2002), hep-lat/0209137

[17] K. Orginos, T. Blum, S. Ohta, Phys. Rev. D73, 094503 (2006), hep-lat/0505024

[18] D. Galletly et al. (QCDSF), PoS LAT2005, 363 (2006), hep-lat/0510050

[19] S. Ohta, K. Orginos (RBCK), Nucl. Phys. Proc. Suppl. 129, 296 (2004), hep-lat/0309113

[20] H.W. Lin, S. Ohta (2006), hep-lat/0610028

[21] D. Toussaint, K. Orginos (MILC), Nucl. Phys. Proc. Suppl. 73, 909 (1999), hep-lat/9809148

[22] K. Orginos, D. Toussaint, R.L. Sugar (MILC), Phys. Rev. D60, 054503 (1999), hep-lat/9903032

[23] C.W. Bernard et al. (MILC), Phys. Rev. D61, 111502 (2000), hep-lat/9912018

[24] C.W. Bernard et al., Phys. Rev. D64, 054506 (2001), hep-lat/0104002 
[25] C. Aubin et al. (MILC), Phys. Rev. D70, 114501 (2004), hep-lat/0407028

[26] D.B. Renner et al. (LHP), Nucl. Phys. Proc. Suppl. 140, 255 (2005), hep-lat/0409130

[27] R.G. Edwards et al. (LHPC), Proc. Sci. LAT2005, 056 (2005), hep-lat/0509185

[28] R.G. Edwards et al. (LHPC), Phys. Rev. Lett. 96, 052001 (2006), hep-lat/0510062

[29] R.G. Edwards et al., Proc. Sci. LAT2006 (2006), hep-lat/0610007

[30] S.R. Beane, P.F. Bedaque, K. Orginos, M.J. Savage (NPLQCD), Phys. Rev. D73, 054503 (2006), hep-lat/0506013

[31] S.R. Beane, P.F. Bedaque, K. Orginos, M.J. Savage, Phys. Rev. Lett. 97, 012001 (2006), hep-lat/0602010

[32] S.R. Beane, K. Orginos, M.J. Savage (2006), hep-lat/0604013

[33] S.R. Beane, K. Orginos, M.J. Savage (2006), hep-lat/0605014

[34] S.R. Beane, P.F. Bedaque, K. Orginos, M.J. Savage (2006), hep-lat/0606023

[35] S.R. Beane et al. (2006), hep-lat/0607036

[36] O. Bar, C. Bernard, G. Rupak, N. Shoresh, Phys. Rev. D72, 054502 (2005), hep-lat/0503009

[37] J.W. Chen, D. O'Connell, R.S. Van de Water, A. Walker-Loud (2005), hep-lat/0510024

[38] S.R. Sharpe, Proc. Sci. LAT2006 (2006), hep-lat/0610094

[39] Y. Shamir (2006), hep-lat/0607007

[40] C. Bernard, M. Golterman, Y. Shamir, Phys. Rev. D73, 114511 (2006), hep-lat/0604017

[41] C. Bernard, Phys. Rev. D73, 114503 (2006), hep-lat/0603011

[42] M. Hasenbusch, Phys. Lett. B519, 177 (2001), hep-lat/0107019

[43] M.J. Peardon, J. Sexton (TrinLat), Nucl. Phys. Proc. Suppl. 119, 985 (2003), hep-lat/0209037

[44] M.A. Clark, A.D. Kennedy, Nucl. Phys. Proc. Suppl. 129, 850 (2004), hep-lat/0309084

[45] M.A. Clark, A.D. Kennedy, Z. Sroczynski, Nucl. Phys. Proc. Suppl. 140, 835 (2005), hep-lat/0409133

[46] M. Luscher, Comput. Phys. Commun. 165, 199 (2005), hep-lat/0409106

[47] W. Kamleh, M.J. Peardon (TrinLat), PoS LAT2005, 106 (2006)

[48] K. Jansen, A. Shindler, C. Urbach, U. Wenger, PoS LAT2005, 118 (2006), hep-lat/0510064

[49] C. Urbach, K. Jansen, A. Shindler, U. Wenger, Comput. Phys. Commun. 174, 87 (2006), hep-lat/0506011

[50] M.A. Clark, Proc. Sci. LAT2006 (2006), hep-lat/0610048

[51] K. Jansen, M. Papinutto, A. Shindler, C. Urbach, I. Wetzorke (XLF), Phys. Lett. B619, 184 (2005), hep-lat/0503031

[52] F. Farchioni et al., Eur. Phys. J. C47, 453 (2006), hep-lat/0512017

[53] K. Jansen, C. Urbach (ETM), Proc. Sci. LAT2006 (2006), hep-lat/0610015

[54] T. Chiarappa et al. (2006), hep-lat/0606011 
[55] A.M. Abdel-Rehim, R. Lewis, Phys. Rev. D71, 014503 (2005), hep-lat/0410047

[56] A.M. Abdel-Rehim, R. Lewis, R.M. Woloshyn, J.M.S. Wu (2006), hep-lat/0610090

[57] A.M. Abdel-Rehim, R. Lewis, R.M. Woloshyn, J.M.S. Wu, Phys. Rev. D74, 014507 (2006), hep-lat/0601036

[58] S. Capitani et al., Phys. Lett. B639, 520 (2006), hep-lat/0511013

[59] W. Detmold, C.J.D. Lin, Phys. Rev. D73, 014501 (2006), hep-lat/0507007

[60] M. Gockeler et al., Nucl. Phys. B717, 304 (2005), hep-lat/0410009

[61] M. Gockeler et al. (2006), hep-lat/0605002

[62] M. Ioannou, H. Panagopoulos, Phys. Rev. D73, 054507 (2006), hep-lat/0601020

[63] B. Bistrovic, Ph.D. thesis, MIT (2005)

[64] S. Capitani (2006), hep-lat/0606022

[65] M. Gockeler et al., Nucl. Phys. B544, 699 (1999), hep-lat/9807044

[66] M. Gockeler et al. (1999), hep-ph/9909253

[67] M. Gockeler et al., Phys. Rev. D72, 054507 (2005), hep-lat/0506017

[68] W.M. Yao et al., Journal of Physics G 33, 1+ (2006), http://pdg.lbl.gov

[69] S. Sasaki, K. Orginos, S. Ohta, T. Blum, Phys. Rev. D68, 054509 (2003), hep-lat/0306007

[70] A.A. Khan et al., Nucl. Phys. Proc. Suppl. 140, 408 (2005), hep-lat/0409161

[71] A.A. Khan et al. (2006), hep-lat/0603028

[72] S.R. Beane, M.J. Savage, Phys. Rev. D70, 074029 (2004), hep-ph/0404131

[73] V. Bernard, U.G. Meissner (2006), hep-lat/0605010

[74] S. Ohta, K. Orginos (RBCK), Nucl. Phys. Proc. Suppl. 140, 396 (2005), hep-lat/0411008

[75] D. Dolgov et al. (LHPC), Phys. Rev. D66, 034506 (2002), hep-lat/0201021

[76] M. Deka et al., Talk given in Lattice 2006 (2006)

[77] M. Gockeler et al., Phys. Rev. D53, 2317 (1996), hep-lat/9508004

[78] M. Gockeler et al., Phys. Lett. B414, 340 (1997), hep-ph/9708270

[79] M. Gockeler et al., Phys. Rev. D63, 074506 (2001), hep-lat/0011091

[80] W. Detmold, W. Melnitchouk, J.W. Negele, D.B. Renner, A.W. Thomas, Phys. Rev. Lett. 87, 172001 (2001), hep-lat/0103006

[81] D. O’Connell, Proc. Sci. LAT2006 (2006), hep-lat/0609046

[82] D. Arndt, M.J. Savage, Nucl. Phys. A697, 429 (2002), nucl-th/0105045

[83] J.W. Chen, X.d. Ji, Phys. Lett. B523, 107 (2001), hep-ph/0105197

[84] J.W. Chen, M.J. Savage, Nucl. Phys. A707, 452 (2002), nucl-th/0108042

[85] C.E. Hyde-Wright, K. de Jager, Ann. Rev. Nucl. Part. Sci. 54, 217 (2004), nucl-ex/0507001

[86] J. Arrington, Phys. Rev. C68, 034325 (2003), nucl-ex/0305009 
[87] L. Andivahis et al., Phys. Rev. D50, 5491 (1994)

[88] M.E. Christy et al. (E94110), Phys. Rev. C70, 015206 (2004), nucl-ex/0401030

[89] I.A. Qattan et al., Phys. Rev. Lett. 94, 142301 (2005), nucl-ex/0410010

[90] M.K. Jones et al. (Jefferson Lab Hall A), Phys. Rev. Lett. 84, 1398 (2000), nucl-ex/9910005

[91] O. Gayou et al. (Jefferson Lab Hall A), Phys. Rev. Lett. 88, 092301 (2002), nucl-ex/0111010

[92] V. Punjabi et al., Phys. Rev. C71, 055202 (2005), nucl-ex/0501018

[93] R.G. Arnold, C.E. Carlson, F. Gross, Phys. Rev. C23, 363 (1981)

[94] P.A.M. Guichon, M. Vanderhaeghen, Phys. Rev. Lett. 91, 142303 (2003), hep-ph/0306007

[95] Y.C. Chen, A. Afanasev, S.J. Brodsky, C.E. Carlson, M. Vanderhaeghen, Phys. Rev. Lett. 93, 122301 (2004), hep-ph/0403058

[96] A.V. Afanasev, S.J. Brodsky, C.E. Carlson, Y.C. Chen, M. Vanderhaeghen, Phys. Rev. D72, 013008 (2005), hep-ph/0502013

[97] M. Göckeler et al. (QCDSF/UKQCD), Proc. Sci. LAT2006 (2006)

[98] C. Alexandrou, G. Koutsou, J.W. Negele, A. Tsapalis (2006), hep-lat/0605017

[99] J.J. Kelly, Phys. Rev. C70, 068202 (2004)

[100] G.V. Dunne, A.W. Thomas, S.V. Wright, Phys. Lett. B531, 77 (2002), hep-th/0110155

[101] M. Gockeler et al. (QCDSF), Phys. Rev. D71, 034508 (2005), hep-lat/0303019

[102] C. Alexandrou, T. Leontiou, J.W. Negele, A. Tsapalis, Proc. Sci. LAT2006 (2006), hep-lat/0610107

[103] C. Alexandrou et al., Phys. Rev. Lett. 94, 021601 (2005), hep-lat/0409122

[104] K.A. Aniol et al. (HAPPEX), Phys. Rev. Lett. 96, 022003 (2006), nucl-ex/0506010

[105] K.A. Aniol et al. (HAPPEX), Phys. Lett. B635, 275 (2006), nucl-ex/0506011

[106] D.S. Armstrong et al. (G0), Phys. Rev. Lett. 95, 092001 (2005), nucl-ex/0506021

[107] D.T. Spayde et al. (SAMPLE), Phys. Lett. B583, 79 (2004), nucl-ex/0312016

[108] F.E. Maas et al. (A4), Phys. Rev. Lett. 93, 022002 (2004), nucl-ex/0401019

[109] F.E. Maas et al., Phys. Rev. Lett. 94, 152001 (2005), nucl-ex/0412030

[110] R. Lewis, W. Wilcox, R.M. Woloshyn, Phys. Rev. D67, 013003 (2003), hep-ph/0210064

[111] D.B. Leinweber et al., Phys. Rev. Lett. 97, 022001 (2006), hep-lat/0601025

[112] E. Shintani et al., Phys. Rev. D72, 014504 (2005), hep-lat/0505022

[113] E. Shintani et al., PoS LAT2006, 123 (2006), hep-lat/0610022

[114] F. Berruto, T. Blum, K. Orginos, A. Soni, Phys. Rev. D73, 054509 (2006), hep-lat/0512004

[115] F. Berruto, T. Blum, K. Orginos, A. Soni, PoS LAT2005, 010 (2006)

[116] P.G. Harris et al., Phys. Rev. Lett. 82, 904 (1999)

[117] M. Guertler et al., Proc. Sci. LAT2006 (2006)

[118] H.R. Fiebig, W. Wilcox, R.M. Woloshyn, Nucl. Phys. B324, 47 (1989) 
[119] J. Christensen, W. Wilcox, F.X. Lee, L.m. Zhou, Phys. Rev. D72, 034503 (2005), hep-lat/0408024

[120] F.X. Lee, L. Zhou, W. Wilcox, J. Christensen, Phys. Rev. D73, 034503 (2006), hep-lat/0509065

[121] W. Detmold, B.C. Tiburzi, A. Walker-Loud, Phys. Rev. D73, 114505 (2006), hep-lat/0603026

[122] W. Detmold, B.C. Tiburzi, A. Walker-Loud, Proc. Sci. LAT2006 (2006), hep-lat/0610018

[123] M. Gockeler et al. (QCDSF), Nucl. Phys. Proc. Suppl. 140, 399 (2005), hep-lat/0409162

[124] P. Hagler, Phys. Lett. B594, 164 (2004), hep-ph/0404138

[125] P. Hagler et al. (LHPC), Phys. Rev. Lett. 93, 112001 (2004), hep-lat/0312014

[126] M. Gockeler et al. (QCDSF), Phys. Rev. Lett. 92, 042002 (2004), hep-ph/0304249

[127] M. Diehl, T. Feldmann, R. Jakob, P. Kroll, Eur. Phys. J. C39, 1 (2005), hep-ph/0408173

[128] M. Gockeler et al., Proc. Sci. LAT2006 (2006)

[129] M. Gockeler et al., Nucl. Phys. Proc. Suppl. 153, 146 (2006), hep-lat/0512011

[130] M. Diehl, P. Hagler, Eur. Phys. J. C44, 87 (2005), hep-ph/0504175

[131] M. Diehl et al. (QCDSF) (2005), hep-ph/0511032

[132] M. Gockeler et al. (QCDSF), Phys. Lett. B627, 113 (2005), hep-lat/0507001

[133] D.W. Sivers, Phys. Rev. D41, 83 (1990)

[134] D.W. Sivers, Phys. Rev. D43, 261 (1991)

[135] D. Boer, P.J. Mulders, F. Pijlman, Nucl. Phys. B667, 201 (2003), hep-ph/0303034

[136] B. Lampe, E. Reya, Phys. Rept. 332, 1 (2000), hep-ph/9810270

[137] B.W. Filippone, X.D. Ji, Adv. Nucl. Phys. 26, 1 (2001), hep-ph/0101224 